\title{
Strategic Capital Budgeting: Asset Replacement under Market Uncertainty*
}

\author{
Grzegorz Pawlina ${ }^{\dagger}$ and Peter M. Kort ${ }^{\ddagger}$
}

June 7, 2003

\begin{abstract}
In this paper the impact of product market uncertainty on the optimal replacement timing of a production facility is studied. The existing production facility can be replaced by a technologically more advanced and thus more cost-effective one. We take into account strategic interactions among the firms competing in the product market by analyzing the problem in a duopolistic setting. We calculate the value of each firm and show that $i$ ) a preemptive (simultaneous) replacement occurs when the associated sunk cost is low (high), ii) despite the preemption effect uncertainty always raises the expected time to replace, and $\mathrm{iii}$ ) the relationship between the probability of optimal replacement within a given time interval and uncertainty is decreasing for long time intervals and humped for short time intervals. Furthermore it is shown that result ii) carries over to the case where firms have to decide about starting production rather than about replacing existing facilities.

Keywords: capital budgeting, real options, first passage time, product market uncertainty, Cournot duopoly

JEL classification: C61, D81, G31
\end{abstract}

${ }^{*}$ This research was undertaken with support from the European Union's Phare ACE Programme 1997. The content of the publication is the sole responsibility of the authors and in no way represents the views of the Commission or its services. The authors would like to thank Kuno Huisman, Enrico Pennings, two anonymous referees, and participants of the Advances in Game Theory Conference in Hilvarenbeek, EFMA 2002 in London, and the Workshop on Recent Topics in Real Options Valuation in Krems for helpful comments.

${ }^{\dagger}$ Corresponding author. Department of Econometrics \& Operations Research and CentER, Tilburg University, P.O. Box 90153, 5000 LE Tilburg, the Netherlands, email: g.pawlina@uvt.nl, phone: + 31134663178 , fax: +31134663280 .

${ }^{\ddagger}$ Department of Econometrics \& Operations Research, and CentER, Tilburg University, the Netherlands, and Department of Economics, UFSIA University of Antwerp, Belgium, email: kort@uvt.nl. 


\section{Introduction}

The present value of growth opportunities often constitutes a significant part of a firm's value. Myers (1977) claims that "the market value of almost all real assets can be partly attributed to associated call options. That is, the ultimate payoff of almost all assets depends on the future discretionary investment of the firm". Fama and French (2001) estimate that on average $42 \%$ of the corporate value in the mid-1990s can be attributed to growth opportunities. Translated into dollars, this means that the average listed US firm holds a $\$ 410$ million portfolio of growth options. Consequently, proper management of such a portfolio via an appropriately designed capital budgeting process becomes crucial for maximizing shareholder value and satisfying return-on-capital requirements. The recognition of the role of real options in capital budgeting dates back to Kester (1984), Trigeorgis and Mason (1987), Trigeorgis (1988), and Dixit and Pindyck (1995). Predictions of the real option theory has been tested empirically, among others, by Paddock et al. (1988), Quigg (1993), Caballero and Pindyck (1996), Holland et al. (2000), and Bulan (2001).

The extensive process of deregulation taking place in the last decade, combined with a wave of mergers and acquisitions, has resulted in an oligopolistic structure of a large number of sectors. A shift towards such a structure takes place not only in traditional regulated markets (telecommunications, energy, transportation) but also in more competitive industries (fast-moving consumer goods, car manufacturing, pharmaceuticals). Consequently, models designed for optimizing capital budgeting decisions while ignoring competitors may cease to be valid. Imperfect competition in the firm's product market requires that strategic interactions with other firm(s) are taken into account. The gap between capital budgeting and strategic planning has already been recognized by Myers (1987) and has been confirmed by Zingales (2000).

Furthermore, the volatile economic environment that firms face these days calls for an appropriate identification of the sources of uncertainty underlying their real activities. Therefore, there is considerable scope for a structural modeling of the product market, where the relationship between the uncertain factor and the cash flow of the firm is explicitly accounted for. ${ }^{1}$

Keeping these considerations in mind, in the paper we address a number of issues, which we relate to the problem of production facility replacement with a more cost-effective one. The questions we endeavor to answer are the following:

- What is the impact of strategic interactions on optimal capital budgeting strategies?

- How does the demand level that triggers the optimal replacement depend on uncertainty, and on the fact whether the firm is the market leader, has the role of the

\footnotetext{
${ }^{1}$ To our best knowledge, within a dynamic real option model the product market structure in a 2-player game is explicitly modeled only by Perotti and Rossetto (2000), Pennings (2002), and Boyer et al. (2002).
} 
follower, or acts identically as its competitor?

- Is the relationship between the uncertainty and the probability of optimal replacement monotonic?

- How does demand uncertainty affect the optimal threshold corresponding to new market entry?

We consider a continuous-time model in which the firm makes an investment decision under product market uncertainty and imperfect competition. The model follows Smets (1991) and Grenadier (1996) in assuming that $i$ ) there are two identical firms competing in the product market, and $i i$ ) the value of the firm depends on the value of a stochastic process but is otherwise time independent. The payoff functions are derived from the firm's reaction curves in the oligopolistic market. Moreover, we calculate the expected replacement timing and determine the probabilities of making optimal replacement within given time intervals.

Under either perfect competition or a monopolistic market structure, modern theory of investment under uncertainty predicts that the firm will wait with investing if uncertainty is higher (cf. McDonald and Siegel, 1986, and Dixit and Pindyck, 1994, Ch. 2). This is due to the fact that investment is irreversible and the firm has an option to postpone it until some uncertainty is resolved. However, if $(i)$ more than one firm holds the investment opportunity, and $(i i)$ the firm's investment decision directly influences payoffs of its competitor(s), opposite effects of increasing uncertainty with respect to the investment timing can arise. First, increasing uncertainty enhances the value of the option to wait. Second, the value of an early strategic investment (made in order to achieve the first-mover advantage) can significantly increase as well. Huisman and Kort (1999) consider a continuous-time duopoly model with profit uncertainty, i.e. where the profit function is subject to multiplicative random shocks (cf. Smets, 1991, and Grenadier, 1996). They show that the effect of a change in the value of the option to wait on the optimal investment threshold is always stronger than the impact of strategic interactions. This implies a negative relationship between uncertainty of the firm's profit flow and investment. On the contrary, Kulatilaka and Perotti (1998) analyze product market uncertainty and find that, in some cases, higher uncertainty does stimulate investment. The authors consider a two-period setting in which (one of the) duopolistic firms can invest in a cost-reducing technology. Product market uncertainty arises due to random shifts in the demand curve. These shifts are caused by both changes in consumers' tastes and a varying mass of consumers. ${ }^{2}$ The resulting payoff function is convex in the stochastic demand parameter since an increase of demand has a more-than-proportional effect on the

\footnotetext{
${ }^{2}$ Profit uncertainty, such as in Huisman and Kort (1999), and Boyer et al. (2002), is equivalent to demand driven either by the changes in the mass of consumers or by the changes in consumers' tastes under zero marginal cost assumption, respectively. In both cases uncertainty can be expressed as random changes in the slope of the demand function.
} 
realized duopolistic profits (firms are responding to higher demand by increasing both output and price). Using Jensen's inequality, Kulatilaka and Perotti (1998) conclude that higher volatility of the product market can increase the expected profit, which has a positive effect on investment. ${ }^{3}$

The aim of this paper is to reconcile the contradicting results of the existing contributions and to determine the effects of product market uncertainty on investment in a fully dynamic, continuous-time framework. This framework embraces both profit convexity, a feature prevailing in oligopolistic markets, and timing flexibility, inherent to most investment decisions. We begin the analysis by describing the equilibrium strategies that occur in the resulting real option game. We show that, contrary to the models based on profit uncertainty, the type of equilibrium depends on the replacement cost: a unique critical level of the replacement cost exists that separates the regions of preemptive and simultaneous equilibria. Consequently, a reduction in the replacement cost can accelerate replacement not only by lowering the level of demand at which the optimal replacement is made, but also by resulting in a switch from simultaneous (late replacement) to preemptive equilibrium (early replacement). ${ }^{4}$ Furthermore, we prove that the minimal demand level triggering the investment increases with uncertainty for both firms. This result holds both for the case in which the investment is associated with replacing an existing asset and for the case in which the firms have to decide when to start up production. Subsequently, we show that, in expectation, product market uncertainty delays investment. We thus can conclude that the result of Kulatilaka and Perotti (1998) does not carry over to a fully dynamic framework. Finally, we analyze the probability of asset replacement within a given time interval. It turns out that the replacement probability decreases with uncertainty for time intervals that include the optimal time to invest in the deterministic case. For shorter intervals there are two opposite effects which leads to a humped relationship between uncertainty and the probability of replacement (cf. Sarkar, 2000).

The model is presented in Section 2, while the value functions and replacement thresholds are derived in Section 3. Section 4 contains a description of the equilibria and in Section 5 the effect of uncertainty on replacement thresholds is determined. In Section 6 the decision to start production in a new market is analyzed. Section 7 examines how uncertainty influences replacement timing and the probability of investment within a given time interval. Section 8 concludes.

\footnotetext{
${ }^{3}$ Jensen's inequality implies here that convexity of a profit function results in the expected profit being higher than the profit associated with expected demand.

${ }^{4}$ This implies that the type of equilibrium can easily be affected by e.g. the authority. The rule imposed by Germany's telecom regulator enabling six companies which acquired the third generation mobile-phone licenses to share the costs of building a new infrastructure, may serve as an example of such an action. See The Economist, 9th June 2001
} 


\section{Framework of the Model}

Consider a risk-neutral firm that has an investment opportunity to replace its existing production facility with a technologically superior one. The firm operates in a duopoly, in which the following inverse linear demand function holds:

$$
p(t)=A(t)-Q(t)
$$

For each $t \in[0, \infty), p(t)$ is the price of a non-durable good/service offered by the firm and can be interpreted as the instantaneous cash flow per unit sold, $A(t)$ is a measure of the size of the demand, and $Q(t)$ is the total amount of the good supplied to the market. Let $r$ denote the riskless interest rate. Parameter $A(t)$ follows a geometric Brownian motion

$$
d A(t)=\alpha A(t) d t+\sigma A(t) d w(t)
$$

where $\alpha$ is the instantaneous drift parameter, $\sigma$ is the instantaneous standard deviation, $d t$ is the time increment, and $d w(t)$ is the Wiener increment. The uncertainty in the model is described by a complete filtered probability space $\left(\Omega, \mathcal{F},\left\{\mathcal{F}_{t}\right\}_{t \in(0, \infty)}, \mathbb{P}\right)$, where $\Omega$ is the state space, $\mathcal{F}$ is the $\sigma$-algebra representing measurable events, and $\mathbb{P}$ is the actual probability measure. The filtration is the augmented filtration generated by the Brownian motion and satisfies the usual conditions. ${ }^{5}$

The other firm operating in the market is identical to the first, both are profitmaximizers and compete in quantities (à la Cournot). ${ }^{6,7}$ The initial constant marginal cost of supplying a unit of the good is $K$ and implementing the new production facility reduces this cost from $K$ to $k$. In order to start using the new facility, Firm $i, i \in\{1,2\}$, has to incur an irreversible cost $I$. Simple algebraic manipulation results in the following instantaneous

\footnotetext{
${ }^{5}$ A filtration $\left\{\mathcal{F}_{t}\right\}$ satisfies the usual conditions if it is right continuous and $\mathcal{F}_{0}$ contains all the $\mathbb{P}$-null sets in $\mathcal{F}$ (see Karatzas and Shreve, 1991, p. 10).

${ }^{6}$ Quantity competition yields the same output as a two-stage game in which the capacities are chosen first and, subsequently, the firms are competing in prices (see Tirole, 1988, p. 216).

${ }^{7}$ Allowing for a certain level of asymmetry between the firms does not change the firms' strategies nor influences the direction of the impact of model parameters. The only difference is that the firm having the relative advantage (e.g. lower marginal cost) is going to realize positive rent in the outcome of the preemption game (cf. Pawlina and Kort, 2002).
} 
profits, $\pi^{i j}$, of Firm $i$ (the other firm is denoted by $\left.j, j \neq i\right)^{8}$

$$
\begin{aligned}
\pi^{00} & =\frac{1}{9}(A-K)^{2}, \\
\pi^{10} & =\frac{1}{9}(A+K-2 k)^{2}, \\
\pi^{01} & =\frac{1}{9}(A-2 K+k)^{2}, \text { and } \\
\pi^{11} & =\frac{1}{9}(A-k)^{2} .
\end{aligned}
$$

Superscript 1 (0) in $\pi^{i j}$ indicates which firm replaced (did not replace) its production facility, where $i$ indicates the own firm and $j$ the competitor. It is seen immediately that

$$
\pi^{10}>\pi^{11}>\pi^{00}>\pi^{01}
$$

The profit of the only firm which replaced the production facility is higher than in the profit of a firm in a situation where two firms made the replacement. In turn, the latter profit exceeds the profit of symmetric firms operating the existing facility, which is still higher than the profit of the only firm which did not replace its production asset.

Admittedly, the chosen model formulation is one out of many possibilities. We choose the linear demand function in order to be able to make comparisons with the results of the two-period model of Kulatilaka and Perotti (1998). Modeling uncertainty as parallel shifts in the demand curve allows us to exploit the effects of convexity of profits in the stochastic demand parameter. Extensions include a more general demand function, a different type of cost function, and Bertrand competition. This paper can be seen therefore as a first fully dynamic investigation of the impact of product market uncertainty on investment timing.

We summarize the problem by describing the strategy space of the firms and the equilibrium concept. Define a simple strategy of Firm $i(i \in\{1,2\})$ for a subgame starting at $s$ as a tuple of real-value functions $\left(G_{i}(t ; \omega), p_{i}(t ; \omega)\right):[s, \infty) \times \Omega \rightarrow[0,1] \times[0,1]$. For every path $\omega$ of the process $(2), G_{i}(t ; \omega)$ is the cumulative probability of Firm $i$ replacing the asset by time $t ; p_{i}(t ; \omega)$ measures the intensity of atoms in the interval $[t, t+d t]$ (see also Section 4.1) and enables coordination in a situation where replacement is optimal for at least one firm (cf. Thijssen et al., 2002). For all paths $\omega \in \Omega$ it holds that:

(i) $G_{i}(t ; \omega)$ is non-decreasing and right-continuous with left limits,

(ii) $p_{i}(t ; \omega)$ is right differentiable and right-continuous with left limits,

(iii) if $p_{i}(t ; \omega)=0$ and $t=\inf \left\{u \mid p_{i}(u ; \omega)>0\right\}$, then the right derivative of $p_{i}(t ; \omega)$ is positive.

\footnotetext{
${ }^{8}$ We assume that $K \ll A(0)$, so that the probability weighted discount factor associated with the event $\{A(t)<2 K-k\}$ is negligible. Waiving this assumption would not significantly contribute to our results and would be done at the expense of explicit analytical formulae for the optimal investment thresholds (cf. Dixit and Pindyck, 1996, p. 191).
} 
Property $(i)$ ensures that $G_{i}(\cdot)$ is a probability distribution. Properties $(i i)$ and $(i i i)$ are for technical convenience and allow for calculating replacement probabilities and for endogenously determining firms' roles in the limiting case when $p_{i}(t ; \omega)=0$.

The strategy space for Firm $i$ is given by the set $S_{i}=\left\{\left(G_{i}(\cdot), p_{i}(\cdot)\right) \mid G_{i}(\cdot)\right.$ satisfies $(i)$, and $p_{i}(\cdot)$ satisfies $(i i)$ and $\left.(i i i)\right\}$. The strategy space of the game is then $\mathcal{S}=S_{1} \times S_{2}$. To determine the firms' optimal policies we use the subgame perfect equilibrium concept, while the firms' strategies are assumed to satisfy intertemporal consistency and $\alpha$-consistency conditions. ${ }^{9}$

\section{Value Functions and Replacement Thresholds}

In this section we establish the value of the firms and their optimal replacement thresholds. There are three possibilities concerning the timing of Firm i's investment relatively to the decision of the competitor (Firm $j$ ). First, Firm $i$ may invest before Firm $j$ does and, therefore, become the leader. Alternatively, Firm $j$ may invest sooner and Firm $i$ becomes the follower. Finally, the firms may invest simultaneously.

The standard approach used to solve dynamic games is to analyze the problem backwards in time. Consequently, we begin with the optimal strategy of the follower. Then, the decision of the leader is analyzed. Finally, we discuss the case of joint investment.

\subsection{Follower}

Consider the case of the firm that replaces as second (follower). Since the other firm (leader) has already replaced its production facility, the follower's replacement decision is not affected by strategic interactions (the follower chooses its optimal threshold as if the roles of the firms are preassigned). From (5) and (6) it is obtained that after replacing the asset by the leader, the value of the follower at time $t$ equals

$$
\begin{aligned}
& V^{F}(A)=E\left[\int_{t}^{T^{F}} \frac{1}{9}(A(s)-2 K+k)^{2} e^{-r(s-t)} d s\right] \\
& +E\left[\int_{T^{F}}^{\infty} \frac{1}{9}(A(s)-k)^{2} e^{-r(s-t)} d s-I e^{-r\left(T^{F}-t\right)}\right],
\end{aligned}
$$

\footnotetext{
${ }^{9}$ Subgame perfect equilibrium requires that for $\forall s \in \mathbb{R}_{+}$a tuple of simple strategies $\left(\left(G_{1}^{s}, p_{1}^{s}\right),\left(G_{2}^{s}, p_{2}^{s}\right)\right)$ be a Nash equilibrium for the subgame starting at $s$. Intertemporal consistency requires that $G_{i}^{s}(v ; \omega)=G_{i}^{t}(v ; \omega)$ and $p_{i}^{s}(v ; \omega)=p_{i}^{t}(v ; \omega)$ for any $s, t$, and $v$ such that $0 \leq s \leq t \leq v<\infty$ and $v=\inf \{u \mid A(u)=A(v)\}$. A strategy is $\alpha$-consistent when for $p_{i}(t ; \omega) \neq p_{i}\left(t_{-} ; \omega\right)$ the jump in the probability distribution $G_{i}(\cdot ; \omega)$ satisfies

$$
G_{i}(t ; \omega)-G_{i}\left(t_{-} ; \omega\right)=\left(1-G_{i}\left(t_{-} ; \omega\right)\right) \frac{p_{i}(t ; \omega)}{p_{i}(t ; \omega)+p_{j}(t ; \omega)-p_{i}(t ; \omega) p_{j}(t ; \omega)},
$$

i.e. when it equals the probability of Firm $i$ replacing at $t$ (for formal definitions see Fudenberg and Tirole, 1985, p. 393, and Thijssen et al., 2002, p. 9).
} 
where $T^{F}$ is the random stopping time associated with replacing the production facility by the follower. The first row of $(8)$ is the expected discounted cash flow received until replacement. At $T^{F}$ the follower makes the replacement and from now on produces against a lower marginal cost $k$. The expected discounted cash flow after replacement is captured by the second row of $(8)$.

Let us consider the value of the follower before its own replacement and after replacement of the competitor. In investment problems of this type (cf. Dixit and Pindyck, 1996) a threshold value of $A$ exists at which the firm is indifferent between investing and refraining from investment. Consequently, the value of the firm is maximized when replacement of the production facility takes place as soon as $A$ exceeds this threshold value. Using standard dynamic programming methodology (see Dixit and Pindyck, 1996) we arrive at the following Bellman equation

$$
r V^{F}=\frac{1}{2} \sigma^{2} A^{2} \frac{\partial^{2} V^{F}}{\partial A^{2}}+\alpha A \frac{\partial V^{F}}{\partial A}+\pi^{01}
$$

Solving the differential equation (9) subject to the condition that $V(A)$ does not explode in the neighborhood of zero gives

$$
V(A)=\underbrace{C A^{\beta}}_{\text {Value of flexibility }}+\underbrace{\frac{1}{9}\left(\frac{A^{2}}{\varrho}-\frac{2(2 K-k) A}{\delta}+\frac{(2 K-k)^{2}}{r}\right)}_{\text {PV of expected cash flow }},
$$

where $C$ is a constant, $\beta$ is the positive root of the equation (cf. Dixit and Pindyck, 1996):

$$
\frac{1}{2} \sigma^{2} \beta(\beta-1)+\alpha \beta-r=0,
$$

and

$$
\begin{aligned}
\varrho & \equiv r-2 \alpha-\sigma^{2} \\
\delta & \equiv r-\alpha .
\end{aligned}
$$

From (10) it can be seen that there are two components contributing to the value of the firm. The first component corresponds to the value of the flexibility to replace the production facility. The remainder of the RHS of (10) reflects the present value of the expected cash flow given that the firm produces with the existing technology forever. Parameters $\delta$ and $\varrho$ can be interpreted as the effective discount rates corresponding to the quadratic and linear component of the profit function, respectively. ${ }^{10}$ Consequently, a finite valuation is obtained if and only if the condition $r-2 \alpha-\sigma^{2}>0$ is satisfied. ${ }^{11}$

\footnotetext{
${ }^{10}$ See Dixit (1993), p. 13-14, for the discusion on the discounting of different powers of the geometric Brownian motion.

${ }^{11}$ In order to assess how restrictive the condition $r-2 \alpha-\sigma^{2}>0$ is, we calculate the maximum feasible growth rate of demand using the parameters of a representative US Standard and Poor's 500 firm (using
} 
To derive the optimal replacement threshold we apply the value-matching and smoothpasting conditions to (10) and the value of the firm after the replacement net of the investment cost. $^{12}$ This leads to

$$
\begin{aligned}
C A^{\beta} & =\frac{1}{9}\left(\frac{4(K-k) A}{\delta}-\frac{4 K(K-k)}{r}\right)-I, \\
\beta C A_{t}^{\beta-1} & =\frac{4}{9} \frac{K-k}{\delta} .
\end{aligned}
$$

From (14) and (15) we obtain the optimal replacement threshold of the follower:

$$
A^{F}=\frac{\beta}{\beta-1} \frac{I+\frac{4 K}{9 r}(K-k)}{\frac{4}{9}(K-k)} \delta .
$$

The optimal time of replacement made by the follower is denoted by

$$
T^{F}=\inf \left\{t \mid A(t) \geq A^{F}\right\}
$$

Note that the optimal threshold (16) increases with uncertainty (through $\beta$ ), and decreases with the growth rate of demand (through $\beta$ and $\delta$ ). The value of the follower can now be calculated by substituting $C$, as derived from (14) and (15), into (10). Such a substitution yields

$$
V^{F}(A)=\left\{\begin{array}{cl}
\frac{1}{9}\left(\frac{A^{2}}{\varrho}-\frac{2(2 K-k) A}{\delta}+\frac{(2 K-k)^{2}}{r}\right) & \text { if } A \leq A^{F} \\
+\left(\frac{1}{9}\left(\frac{4(K-k) A^{F}}{\delta}-\frac{4 K(K-k)}{r}\right)-I\right)\left(\frac{A}{A^{F}}\right)^{\beta} & \text { if } \quad A>A^{F} \\
\frac{1}{9}\left(\frac{A^{2}}{\varrho}-\frac{2 k A}{\delta}+\frac{k^{2}}{r}\right)-I & \text { if }
\end{array}\right.
$$

\subsection{Leader}

Having established the optimal replacement rule of the follower, we are ready to determine the payoff of the firm that invests as the leader. The value function of the leader, historical CRSP data from 1926-99, as reported in Bodie et al., 2002). By applying Itô's lemma one can show that the volatility of the process proportional to the square of the original process equals two times the volatility of the original process. Hence, since the value of the firm is proportional to $A^{2}$, its instantaneous volatility equals twice the volatility of $A$. As a consequence, for a standard deviation of $20.2 \%$, which is typical for the representative S\&P 500 firm, the implied $\sigma$ equals $10.1 \%$. Given that the average yield on long-term treasury bonds amounts in the relevant period to $5.36 \%$, finite valuations are ensured for values of parameter $\alpha$ ranging from minus infinity to $\left(0.0536-(0.101)^{2}\right) / 2=2.17 \%$. This corresponds to the $4.39 \%\left(1.0217^{2}-1\right)$ growth of demand measured as the consumers' valuations integrated over the mass of consumers.

${ }^{12}$ The value matching condition equalizes the value of the firm before the replacement (including the replacement option), as in (10), and the value after the replacement net of the associated sunk cost. Upon observing that the value after the replacement corresponds to the expected cash flow from new assets in place and equals $\frac{1}{9}\left(\frac{A^{2}}{\varrho}-\frac{2 k A}{\delta}+\frac{k^{2}}{r}\right)$, condition (14) is obtained. Condition (15) is obtained by taking the derivatives of (14) with respect to $A$. 
evaluated at the moment of investing, $t$, is

$$
V^{L}(A)=E\left[\int_{t}^{T^{F}} \frac{1}{9}(A(s)+K-2 k)^{2} e^{-r(s-t)} d s-I+\int_{T^{F}}^{\infty} \frac{1}{9}(A(s)-k)^{2} e^{-r(s-t)} d s\right] .
$$

The first two components of (19) correspond to the present value of the leader's profits realized until the moment of the follower's investment, net of the leader's sunk cost. The second integral corresponds to the discounted perpetual stream of profits obtained after the investment of the follower.

Analogous to expression (18) of the follower problem, we can express the value of Firm $i$ as the leader in the following way:

$$
V^{L}(A)=\left\{\begin{array}{ccc}
\frac{1}{9}\left(\frac{A^{2}}{\varrho}+\frac{2(K-2 k) A}{\delta}+\frac{(K-2 k)^{2}}{r}\right)-I & \text { if } & A \leq A^{F}, \\
-\frac{1}{9}\left(\frac{2(K-k) A^{F}}{\delta}+\frac{(K-2 k)^{2}-k^{2}}{r}\right)\left(\frac{A}{A^{F}}\right)^{\beta} & & \\
\frac{1}{9}\left(\frac{A^{2}}{\varrho}-\frac{2 k A}{\delta}+\frac{k^{2}}{r}\right)-I & \text { if } & A>A^{F} .
\end{array}\right.
$$

The first row of (20) corresponds to the net present value of the leader profits without the follower ever replacing its asset. The second row reflects the present value of future profits lost due to the follower's replacement at $A^{F}$. This loss is caused by the fact that after the follower has invested, the follower can produce in a cheaper way, which makes it a stronger competitor for a leader. Consequently, the value of the leader is positively related to $A^{F}$. The last row represents the net present value of profits in a situation where it is optimal for the follower to invest immediately.

\subsection{Simultaneous Investment}

It is possible that the firms decide to invest simultaneously. The time- $t$ value function of both firm investing optimally at the joint investment threshold is

$$
\begin{aligned}
V^{S}(A)= & E\left[\int_{t}^{T^{S}} \frac{1}{9}(A(s)-K)^{2} e^{-r(s-t)} d s-I e^{-r\left(T^{S}-t\right)}\right] \\
& +E\left[\int_{T^{S}}^{\infty} \frac{1}{9}(A(s)-k)^{2} e^{-r(s-t)} d s\right],
\end{aligned}
$$

where

$$
T^{S}=\inf \left\{t \mid A(t) \geq A^{S}\right\}
$$

and

$$
A^{S}=\frac{\beta}{\beta-1} \frac{I+\frac{1}{9} \frac{K^{2}-k^{2}}{r}}{\frac{2}{9}(K-k)} \delta
$$


Expression (21) can be interpreted analogous to (8) and (19). Consequently, the value of Firm $i$ when the investment is made simultaneously equals

$$
V^{S}(A)=\left\{\begin{array}{ccc}
\frac{1}{9}\left(\frac{A^{2}}{\varrho}-\frac{2 K A}{\delta}+\frac{K^{2}}{r}\right) & \text { if } & A \leq A^{S}, \\
+\left(\frac{1}{9}\left(\frac{2(K-k) A^{S}}{\delta}-\frac{K^{2}-k^{2}}{r}\right)-I\right)\left(\frac{A}{A^{S}}\right)^{\beta} & \text { if } & \\
\frac{1}{9}\left(\frac{A^{2}}{\varrho}-\frac{2 k A}{\delta}+\frac{k^{2}}{r}\right)-I & \text { if } & A>A^{S} .
\end{array}\right.
$$

The last row equals the value of the firm when the simultaneous investment is made immediately. In such a case, the value of the firm is denoted by $V^{J}(A)$.

\section{Equilibria}

Since both firms are ex ante identical, it is natural to consider symmetric replacement strategies and assume the firms' roles being endogenous, i.e. that it is not determined beforehand which firm will be the first to replace. There are two types of equilibria that can occur under this choice of strategies. We start by presenting the preemptive equilibrium, which is followed by a description of the simultaneous equilibrium.

\subsection{Preemptive Equilibrium}

The first type of equilibrium is a preemptive equilibrium where Firm $i$ is the leader and Firm $j$ is the follower. Let us define $A^{P}$ to be the root of

$$
\xi(A)=V^{L}(A)-V^{F}(A)
$$

on the interval $\left(0, A^{F}\right)$. Assume for the moment that $A(0)<A^{P}$. Since on the interval $\left(A^{P}, A^{F}\right)$ the payoff of the leader is higher than the payoff of the follower, each firm will have an incentive to be the leader at the moment that $A(t) \in\left(A^{P}, A^{F}\right)$. In the search for an equilibrium we reason backwards in terms of the values of $A$ (note that equation (2) does not imply that $A$ increases monotonically over time). Consider a value of $A$ such that $A \in\left(A^{P}, A^{F}\right)$. Then it holds that the leader's payoff is higher than the payoff of the follower. This implies that (without loss of generality) Firm $i$ has an incentive to be the first investor there. Firm $j$ anticipates this and would invest at $A-\varepsilon$. Repeating this reasoning we reach an equilibrium in which Firm $i$ invests at $A^{P}$ and Firm $j$ waits with replacement until demand equals $A^{F}$. Note that if both firms invest at $A^{P}$ with probability one, they end up with the low payoff $V^{J}\left(A^{P}\right)$. At $A=A^{P}$ simultaneous replacement is not profitable because demand is insufficient. Figure 1 depicts the payoffs (relative to the follower payoff) associated with the preemptive equilibrium.

Since the firms are identical it is not clear beforehand which of them will be the leader. First, we present the endogenous selection mechanism according to which the roles 


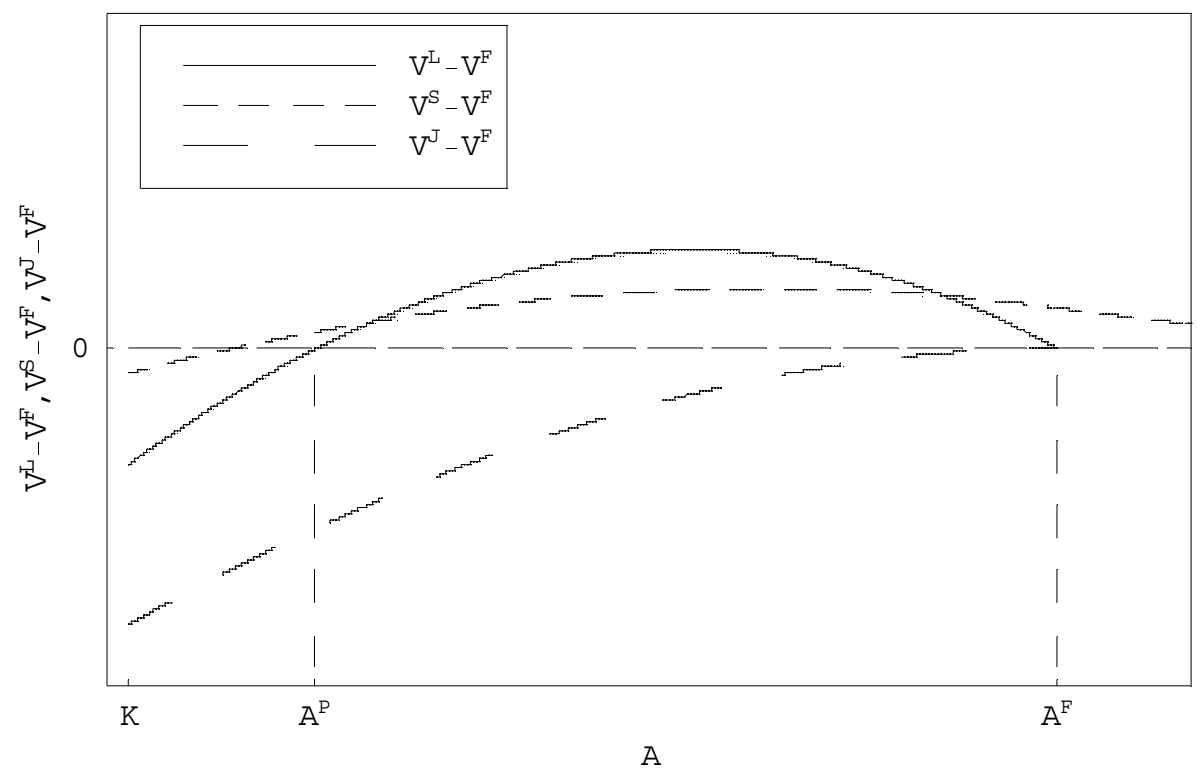

Figure 1: The values of the leader, $V^{L}$, optimal simultaneous replacement, $V^{S}$, and early simultaneous replacement, $V^{J}$, relative to the value function of the follower, $V^{F}$, for a set of parameter values resulting in a preemptive replacement at $A^{P}$ (leader) and $A^{F}$ (follower).

of the firms are determined. Later, we discuss how the roles of the firms can be determined in real-life situations.

In order to formalize the analysis of how the roles of the firms are determined, we adopt the approach of Thijssen et al. (2002). This approach extends the perfect equilibrium concept of Fudenberg and Tirole (1985) to stochastic games. As in Fudenberg and Tirole (1985), the firms use mixed strategies in which the expected payoff is equal to the payoff of the follower (recall that the firms are risk-neutral). It is argued there (see also Torvund, 1999) that in continuous-time preemption games a closed loop strategy of Firm $i$ consists of a collection of simple strategies $\left(G_{i}(\cdot), p_{i}(\cdot)\right)$. $G_{i}(t)$ is the probability that Firm $i$ has invested by time $t$. The function $p_{i}(t)$ is the measure of the intensity of atoms in the interval $[t, t+d t]$. It can be interpreted as the probability of playing the first row and the first column (for Firm 1 and Firm 2, respectively) in the following $2 \times 2$ game: $\{\{$ replace, replace $\},\{$ replace, don't replace $\},\{$ don't replace, replace $\},\{$ don't replace, don't replace $\}$. Playing this game costs no time and the game is repeated until at least one firm invests.

In case $A \in\left(A^{P}, A^{F}\right)$, the value of $p_{i}\left(p_{j}\right)$ is determined as follows. Since $p_{i}\left(p_{j}\right)$ is the probability that Firm $i(j)$ replaces its asset, Firm $i$ sets $p_{i}$ such that

$$
V_{i}=\max _{p_{i}}\left[p_{i}\left(1-p_{j}\right) V^{L}+\left(1-p_{i}\right) p_{j} V^{F}+p_{i} p_{j} V^{J}+\left(1-p_{i}\right)\left(1-p_{j}\right) V_{i}\right] .
$$

Since Firm $i$ replaces its asset with probability $p_{i}$ and Firm $j$ with probability $p_{j}$, the 
probability that Firm $i$ obtains the leader role is $p_{i}\left(1-p_{j}\right)$. Similarly, with probability $\left(1-p_{i}\right) p_{j}$ Firm $i$ is the follower, $p_{i} p_{j}$ is the joint investment probability, and with probability $\left(1-p_{i}\right)\left(1-p_{j}\right)$ nothing happens and the game is repeated. After writing down the first-order conditions for Firm $i$ and Firm $j$, and imposing symmetric strategies, we obtain that

$$
p=p_{i}=p_{j}=\frac{V^{L}-V^{F}}{V^{L}-V^{J}} .
$$

It holds that the equilibrium strategy of Firm $i$ equals

$$
p(t)=\left\{\begin{array}{lll}
0 & \text { if } & A(t)<A^{P} \\
\frac{V^{L}(A(t))-V^{F}(A(t))}{V^{L}(A(t))-V^{J}(A(t))} & \text { if } & A(t) \in\left[A^{P}, A^{F}\right] \\
1 & \text { if } \quad A(t)>A^{F}
\end{array}\right.
$$

and

$$
G(t)=\left\{\begin{array}{lll}
0 & \text { if } \quad A(t)<A^{P}, \\
\frac{V^{L}(A(t))-V^{J}(A(t))}{V^{L}(A(t))-2 V^{J}(A(t))+V^{F}(A(t))} & \text { if } & A(t) \in\left[A^{P}, A^{F}\right) . \\
1 & \text { if } & A(t) \geq A^{F}
\end{array}\right.
$$

After substituting $p=p_{i}=p_{j}$ in (26), the value of Firm $i$ can be expressed as

$$
V_{i}=\frac{p(1-p) V^{L}+p(1-p) V^{F}+p^{2} V^{J}}{2 p-p^{2}}=V^{F}
$$

Consequently, for $A(t) \in\left[A^{P}, A^{F}\right]$, the probability that one of the firms invests at time $t$ equals

$$
\operatorname{Pr}(\text { one firm has invested } \mid t)=\frac{2-2 p(t)}{2-p(t)}
$$

while the firms invest simultaneously with probability

$$
\operatorname{Pr}(\text { two firms have invested } \mid t)=\frac{p(t)}{2-p(t)} \text {. }
$$

If $A(0)<A^{P}$, the leader payoff curve lies below the follower curve which implies that it is optimal for both firms to refrain from investment. At $A=A^{P}$, the leader and the follower values are equal. This implies that (28) and (31) yield the probability of being the leader (or follower) equal to $\frac{1}{2}$. The probability of simultaneous investment at $A=A^{P}$ is therefore equal to zero. The leader invests at the moment that $A=A^{P}$, which is the smallest solution of $V^{L}(A)=V^{F}(A)$, and the follower waits until $A^{F}$ is reached.

If the stochastic process starts at $A(0) \geq A^{P}$, at least one of the firms invests immediately. The probability of an immediate joint investment leading to the low payoff $V^{J}(A(0))$ is $\frac{p(0)}{2-p(0)}$ (cf. (32)). In this case, according to $(28), p(0)>0$ since the payoff of the leader exceeds the payoff of the follower. This makes the probability of investing jointly, and ending up with a low payoff of $V^{J}(A(0))$, become positive. 
In order to be able to translate the derived mixed strategies into applicable decision rules (since "real-world decision makers do not flip coins"), we refer to the approach of Harsanyi (1973). He has shown that a mixed-strategy equilibrium of a complete information game, such as the one analyzed in this paper, can be interpreted as the limit of a pure-strategy equilibrium of a slightly perturbed game of incomplete information (see also Tirole, 1988). ${ }^{13}$ Consequently, instead of assuming that firms play mixed strategies in the described above $2 \times 2$ game, one can assume that the actual payoff resulting from becoming the leader equals $V^{L}(A(t))+\theta$, and $\theta$ is distributed according to a density function $\varphi(\theta)$ with a bounded support $[\underline{\theta}, \bar{\theta}], \underline{\theta}<0<\bar{\theta}$. ${ }^{14}$ The firm observes its own realization of $\theta$ but not the one of its competitor. Now, it can be shown that a symmetric Bayesian equilibrium in pure strategies exists. There is a critical value of $\theta=\theta^{*}$ such that the optimal strategy for Firm $i$ is to invest if and only if $\theta_{i}>\theta^{*}$. Consequently, the firms do not have to invoke randomizing devices in the implementation of optimal actions. ${ }^{15}$

\subsection{Simultaneous Equilibrium}

The other type of outcome that can occur in the analyzed real option game is the simultaneous replacement equilibrium. In such a case, both firms replace their production facilities at $T^{S}$ (see (22)). A graphical illustration of the simultaneous equilibrium is depicted in Figure 2. From this figure it can be concluded that no firm has an incentive to deviate from this equilibrium since the payoff of this strategy exceeds all other payoffs. ${ }^{16}$

The occurrence of a particular type of equilibrium is determined by the relative payoffs. The preemptive equilibrium occurs when

$$
\exists A \in\left(A^{P}, A^{F}\right) \text { such that } V^{L}(A)>V^{S}(A),
$$

i.e. when for some $A$ it is more profitable to become the leader than to replace produc-

\footnotetext{
${ }^{13}$ For sufficient conditions on the payoff functions and information structure when such an interpretation is possible, see Milgrom and Weber (1986).

${ }^{14}$ Here, the uncertainty about the value of parameter $\theta$ is just a reduced form representation of uncertainty about the value of (the one of) the firm-specific primitive parameters of the model, such as a unit production cost, replacement cost or demand elasticity.

${ }^{15}$ An interesting example of a practical solution to the coordination problem is presented in Hoppe and Lehmann-Grube (2001) in a context of computer fairs: "If both firms plan to make an announcement at the same fair, one firm happens to have its press conference before the other with probability $1 / 2$. The other firm observes the announcement of the first firm, and may decide to postpone its introduction date to a later fair".

${ }^{16}$ Of course, the payoffs resulting from the preemptive equilibrium in Section 4.1 may be lower than those associated with the optimal joint replacement. However, the occurrence of the preemptive equilibrium, as in Section 4.1, is due to the fact that values of $A$ exist that the corresponding leader payoff exceeds the value from the joint replacement strategy. It is the lack of coordination among the firms (with possible transfer of excess value) that leads to ex post Pareto-inefficient outcomes. In the case of the simultaneous equilibrium the payoff of the leader never exceeds the payoff from optimal joint replacement and therefore the preemptive equilibrium, while still existent, is Pareto-dominated (see Fudenberg and Tirole, 1985)
} 


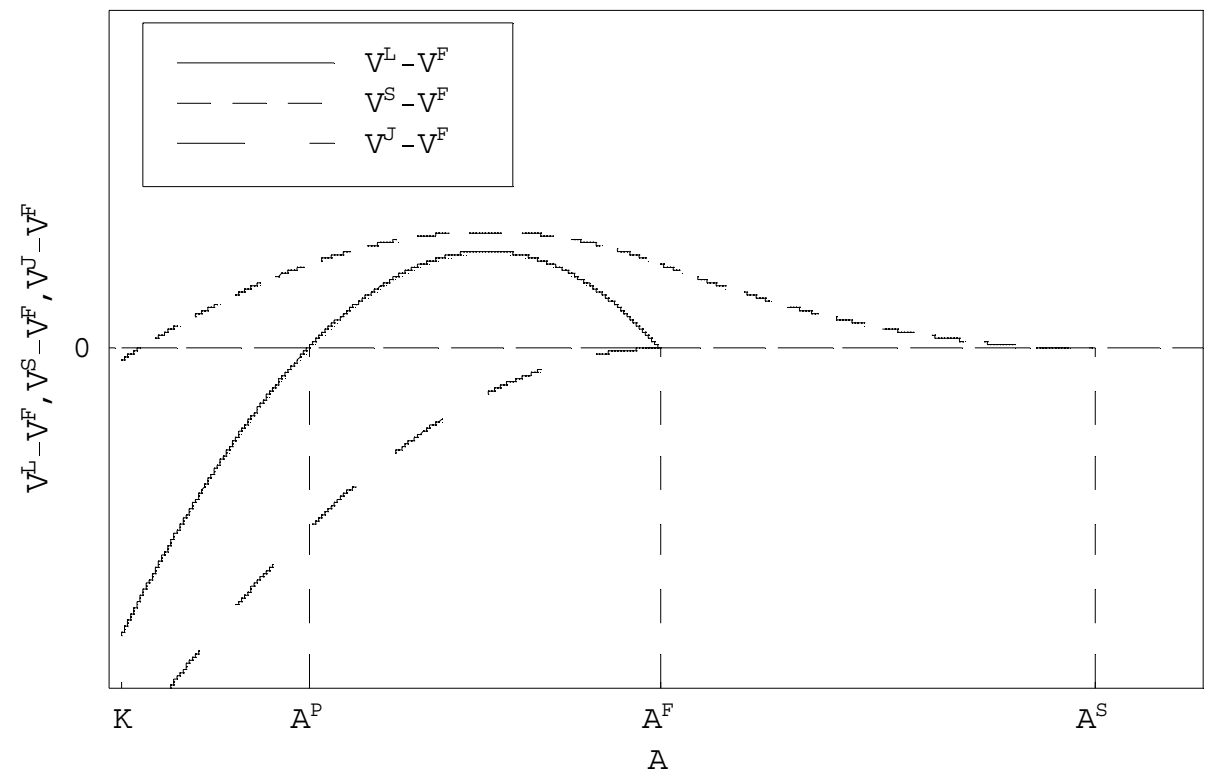

Figure 2: The values of the leader, $V^{L}$, optimal simultaneous replacement, $V^{S}$, and early simultaneous replacement, $V^{J}$, relative to the value function of the follower, $V^{F}$, for a set of parameter values resulting in the optimality of a simultaneous replacement at $A^{S}$.

tion facilities simultaneously. Otherwise, simultaneous replacement is the Pareto-dominant equilibrium. In the latter case, the strategy of Firm $i$ can be formalized as

$$
p(t)=\left\{\begin{array}{lll}
0 & \text { if } & A(t)<A^{S} \\
1 & \text { if } & A(t) \geq A^{S}
\end{array}\right.
$$

and

$$
G(t)=\left\{\begin{array}{lll}
0 & \text { if } & A(t)<A^{S} \\
1 & \text { if } & A(t) \geq A^{S}
\end{array}\right.
$$

The following proposition implies that firms replace their production facilities simultaneously if the investment cost is sufficiently high.

Proposition $1 \quad A$ unique $I^{*}$ exists such that $\forall I>I^{*}$ simultaneous replacement is the Pareto-dominant equilibrium.

Proof. See Appendix A.

For a sufficiently low replacement cost, i.e. for $I<I^{*}$, it is optimal for any firm to replace its production technology and to become the leader even at the very early stage of the market development. In this case, gaining a (temporary) first-mover advantage justifies an earlier capital spending. In the opposite situation, i.e. when replacement cost exceeds $I^{*}$, firms are better off by utilizing the existing production facility. In that case it is optimal to 
wait with replacement until market significantly improves, even at the cost of foregoing the superior first-mover payoff.

Proposition 1 is an important result with respect to the comparison between the real option exercise game with profit uncertainty and the situation where the firms face product market uncertainty. In the first case the occurrence of either of the equilibria does not depend on the irreversible cost associated with the investment decision (see Huisman and Kort, 1999). This results from the fact that the optimal threshold under profit uncertainty is proportional to the investment cost $I$. This proportionality is a consequence of the multiplicative way in which uncertainty enters the profit function. Conversely, introducing market uncertainty in a Cournot model results in the optimal threshold being no longer proportional to $I$. This is the reason why the resulting equilibrium regions depend on the sunk cost. ${ }^{17}$

It is worth pointing out that Proposition 1 does not imply that firms simultaneously replace their assets only if the replacement cost is sufficiently high. First, consider a reduction in the cost from $I_{1}$ to $I_{2}\left(<I_{1}\right)$ such that $A_{(1)}^{P}>A(0)>A_{(2)}^{P}$, where $A_{(k)}^{P}$ is the preemption threshold corresponding to the cost $I_{k}$. Such a reduction introduces a positive probability of joint replacement in the preemptive equilibrium (cf. equations (28) and (32)). Moreover, a further decrease in the replacement cost results eventually in $A(0)$ being located to the right of the right intersection of functions $V^{L}$ and $V^{S}$, thus in the region where $V^{S}>V^{L}$ (cf. Figure 1). This results in simultaneous investment occurring at $A^{S}$. Eventually, a further decrease makes the replacement cost so low that all the thresholds, including $A^{S}$, lie below $A(0)$. In such a case firms replace their assets simultaneously at $t=0$ and the outcomes of the preemptive and simultaneous equilibria coincide.

\section{Uncertainty and Asset Replacement Thresholds}

Since the firms' decisions to replace production assets are irreversible (sunk cost $I$ cannot be recovered) and they have the possibility of postponing the replacement, they replace their production assets later than a simple NPV rule would indicate. In a non-strategic framework, there exists an option value of waiting for better (but never complete) information which is taken into account before committing the corporate resources. As uncertainty about the demand grows, the firm is going to wait with replacement for a higher level of demand, as the classical real option theory suggests. However, it also has to take into account the interactions in the product market, that may substantially reduce the value of the timing flexibility. Kulatilaka and Perotti (1998) obtained in a two-period model that these interactions may in fact result in a negative relationship between the required level of demand at which resources are committed and uncertainty. In this section, we examine how uncertainty influences the

\footnotetext{
${ }^{17}$ In general, the investment cost affects the boundaries of the equilibrium regions. Therefore, the lack of such a relationship in a profit uncertainty model is rather a coincidence than a rule.
} 
level of demand triggering investment in our continuous-time model.

First, we investigate the impact of volatility on the optimal asset replacement thresholds of the follower and of optimal simultaneous replacement. In these cases the optimal threshold, $A^{\text {opt }}$, can be expressed as

$$
A^{\text {opt }}=\frac{\beta}{\beta-1} f(I, K, k, r, \alpha) .
$$

It is straightforward to derive that

$$
\frac{\partial A^{o p t}}{\partial\left(\sigma^{2}\right)}=-\frac{1}{(\beta-1)^{2}} f(I, K, k, r, \alpha) \frac{\partial \beta}{\partial\left(\sigma^{2}\right)}>0,
$$

so that the optimal replacement thresholds of the follower and of optimal simultaneous replacement increase in uncertainty. In the case of the follower's decision, the competitor has already replaced its asset. Hence, what is left to do for the remaining firm is to choose the optimal replacement timing. Since the opponent has already taken its decision, strategic interactions do not play a role here. So, as in standard real option theory, also here the threshold goes up with uncertainty, which reflects the value of waiting argument. In determining the optimal simultaneous replacement timing strategic interactions do not play a role either. Therefore, analogous to the follower's case, the value of waiting argument also prevails here.

The impact of volatility on the production facility replacement threshold of the leader requires an additional analysis. We set the marginal cost $k$ to zero to simplify the notation. ${ }^{18}$ The replacement threshold of the leader equals max $\left[A(0), A^{P}\right]$, where $A^{P}$ is the smallest root of $\xi(A)=0$. To determine the effect of market uncertainty on $A^{P}$, we calculate the derivative of $\xi(A)$ with respect to $\sigma^{2}$. The change of (25) resulting from a marginal increase in $\sigma^{2}$ can be decomposed as follows

$$
\frac{d \xi(A)}{d\left(\sigma^{2}\right)}=(\underbrace{\frac{\partial \xi(A)}{\partial \beta}}_{\text {"Waiting" effect }}+\underbrace{\frac{\partial \xi(A)}{\partial A^{F}} \frac{d A^{F}}{d \beta}}_{\text {Strategic effect }}) \frac{\partial \beta}{\partial\left(\sigma^{2}\right)} .
$$

The derivative $\frac{\partial \xi(A)}{\partial \beta} \frac{\partial \beta}{\partial\left(\sigma^{2}\right)}$ directly measures the influence of uncertainty on $\xi(A)$, thus on the net benefit of being the leader. The product $\frac{\partial \xi(A)}{\partial A^{F}} \frac{d A^{F}}{d \beta} \frac{\partial \beta}{\partial\left(\sigma^{2}\right)}$ reflects the impact on the net benefit of being the leader of the fact that the follower replacement threshold increases with uncertainty.

\footnotetext{
${ }^{18}$ An additional motivation for this simplification is provided by the fact that for the majority of intangible/information products the marginal cost of a unit of good/service is negligible (cf. Shapiro and Varian, 1998).
} 
It can be shown that

$$
\begin{aligned}
\frac{\partial \xi(A)}{\partial \beta} \frac{\partial \beta}{\partial\left(\sigma^{2}\right)} & <0 \\
\frac{\partial \xi(A)}{\partial A^{F}} \frac{d A^{F}}{d \beta} \frac{\partial \beta}{\partial\left(\sigma^{2}\right)} & >0 .
\end{aligned}
$$

At first sight, the joint impact of both effects is ambiguous. (39) represents the simple value of waiting argument: if uncertainty is large, it is more valuable to wait for new information before replacing the existing production facility (cf. Dixit and Pindyck, 1996). As we have just seen, this also holds for the follower. The implication for the leader of the follower replacing later is that the leader has a cost advantage for a longer time. This makes an earlier replacement of the leader potentially more beneficial. This effect is captured by (40), which can thus be interpreted as an increment in the strategic value of becoming the leader vs. the follower resulting from the delay in the follower's implementation of the superior technology. Obviously, the latter effect is not present in monopolistic/perfectly competitive markets, where the impact of uncertainty is unambiguous.

It is possible to show that the direct effect captured by (39) dominates, irrespective of the values of the input parameters.

Proposition 2 When uncertainty in the product market increases, the threshold value of the demand at which the leader replaces its production facility increases too.

Proof. See Appendix A.

From Proposition 2 it can be concluded that the leader threshold responds to volatility in a qualitatively similar way as a non-strategic threshold, i.e. it increases with uncertainty. The reason for this result is the following. First, in our model we introduced the possibility of postponing the replacement of the production facility. Increased uncertainty raises the profitability of replacement (because the follower replaces later) but, however, the value of the option to wait rises even more. Second, uncertainty could be beneficial for earlier replacement because of the convex shape of the net gain function, resulting in a power option-like type of payoff (cf. Kulatilaka and Perotti, 1998). Then, while performing a mean preserving spread, downside losses are more than compensated by upside gains. However, unlike the two-period framework of Kulatilaka and Perotti (1998), in our continuous-time model the net gain function is always linear in the stochastic variable $A$. If the leader invests, the profit flow $\pi^{00}$ is replaced by the profit flow $\pi^{10}$, and it is clear from (3) and (4) that $\pi^{10}-\pi^{00}$ is linear. The same holds for the follower investment $\left(\pi^{11}-\pi^{01}\right.$ linear $)$ and simultaneous investment (linearity of $\pi^{11}-\pi^{00}$ ). To see whether the convexity argument could also work here, in Section 6 we consider the decision to start production. In this case the firms are not active initially and can start up production only upon investing. Consequently, the net gain flows for the leader, and the follower, being equal to $\pi^{10}$ and $\pi^{01}$, respectively, are convex in A. 


\section{Decision to Start Production}

Consider two firms having the possibility to start production in a new market where there is no incumbent. The new market assumption implies, in contrast with Sections 3-4, that the firms can only start realizing profits after incurring a sunk cost $I$. It still holds that demand follows the stochastic process (2). Again, the marginal cost of a unit of output after starting production, $k$, is assumed to equal zero.

First, we calculate the value of the demand parameter for which it is optimal for the follower to start production. After, by now, familiar steps it is obtained that

$$
A^{F N}=3 \sqrt{\frac{\beta}{\beta-2} I \varrho} .
$$

Given that for positive $\varrho$ it holds that $\beta>2$, it is straightforward to show that

$$
\frac{\partial A^{F N}}{\partial\left(\sigma^{2}\right)}>0
$$

The optimal follower threshold (41) exists only for $\sigma^{2}<r-2 \alpha$. For a relatively high degree of uncertainty, i.e. for $\sigma^{2} \geq r-2 \alpha$ (which corresponds to $\beta \in(1,2]$ ), the follower will never start production since for such levels of uncertainty the value of the option to invest always exceeds the net present value of investment. In the limiting case, the optimal follower threshold (41) is equal to

$$
\overline{A^{F N}}=\lim _{\sigma^{2} \uparrow r-2 \alpha} A^{F N}=3 \sqrt{(3 r-4 \alpha) I}
$$

(for a derivation see Appendix B). Equation (43) corresponds to the maximal value of $A^{F N}$ provided that it is finite. In case information about the uncertainty level is imperfect, the investment problem is solved by first calculating the uncertainty implied by the threshold $\overline{A^{F N}}$. Subsequently, the decision maker can decide whether the true level of uncertainty is more likely to lie below or above the implied value. In the latter case, he should refrain from entering the market.

Now, let $t$ be the moment at which the leader starts producing in the new market. The value of the follower at $t$ is equal to

$$
V^{F N}(A)=\left\{\begin{array}{cc}
\left(\frac{\frac{1}{9}\left(A^{F N}\right)^{2}}{\varrho}-I\right)\left(\frac{A}{A^{F N}}\right)^{\beta} & \text { if } \quad A \leq A^{F N}, \\
\frac{1}{9} \frac{A^{2}}{\varrho}-I & \text { if } \quad A>A^{F N} .
\end{array}\right.
$$

The value of the leader at $t$ can be expressed as

$$
V^{L N}(A)=\left\{\begin{array}{cc}
\frac{1}{4} \frac{A^{2}}{\varrho}-I & \text { if } \quad A \leq A^{F N} \\
-\frac{5}{36} \frac{\left(A^{F N}\right)^{2}}{\varrho}\left(\frac{A}{A^{F N}}\right)^{\beta} & \text { if } \quad A>A^{F N} \\
\frac{1}{9} \frac{A^{2}}{\varrho}-I & \text { if }
\end{array}\right.
$$


From (44) and (45) it is obtained that indeed the leader and follower values are convex in $A$. The threshold of the leader, being the preemption point, is the smallest solution of the following equation

$$
V^{L N}(A)-V^{F N}(A)=\frac{1}{4} \frac{A^{2}}{\varrho}-I-I\left(\frac{9}{4} \frac{\beta}{\beta-2}-1\right)\left(\frac{A}{A^{F N}}\right)^{\beta}=0 .
$$

The impact of uncertainty on the threshold of the leader is not straightforward. Similar as in the model with the firms initially competing in the product market, there are two effects: the effect of the waiting option and of the strategic option. Let us denote $V^{L N}(A)-V^{F N}(A)$ by $\xi^{N}(A)$. We have

$$
\begin{aligned}
& \frac{d \xi^{N}(A)}{d\left(\sigma^{2}\right)}= \\
& \underbrace{\frac{\partial \xi(A)}{\partial\left(\sigma^{2}\right)}+\frac{\partial \xi(A)}{\partial A^{F N}} \frac{d A^{F N}}{d\left(\sigma^{2}\right)}}_{\text {Discount rate effect }}+\underbrace{\left(\frac{\partial \xi(A)}{\partial \beta}+\frac{\partial \xi(A)}{\partial A^{F N}} \frac{d A^{F N}}{d \beta}\right) \frac{\partial \beta}{\partial\left(\sigma^{2}\right)}}_{\text {Direct uncertainty effect }}
\end{aligned}
$$

Uncertainty affects the magnitude of each of the mentioned effects via parameter $\beta$, as in Section 5 , and via the effective discount rate, $\varrho$ (see (12)). The latter contribution results from the convexity of the profit function, i.e. its proportionality to the square of the underlying stochastic variable $A$ (see (45)).

After substituting the functional forms of $V^{L N}(A)$ and $V^{F N}(A)$ into $\xi^{N}(A)$ and calculating the derivative explicitly, the following result is obtained.

Proposition 3 The threshold value of the demand at which the leader starts production increases in uncertainty.

Proof. See Appendix A.

Analogous to the follower case, there exists a critical level of uncertainty, $\sigma^{2}=r-2 \alpha$, above which it is optimal for the leader never to invest. In the limit, where $\sigma^{2} \rightarrow r-2 \alpha$, the leader threshold is the smaller root of the equation (for the proof see Appendix B)

$$
\left(\left(\frac{A}{A^{F N}}\right)^{2}-1\right) I-\frac{A^{2}}{2(3 r-4 \alpha)} \ln \left(\frac{A}{A^{F N}}\right)=0 .
$$

The conclusion is that also in the case of a new market, uncertainty raises the threshold levels of market demand at which it is optimal for firms to invest. Moreover, the resulting convexity of the payoff functions not only raises the threshold of the firms but also results in a subset of parameters for which no replacement is optimal. 


\section{$7 \quad$ Uncertainty and Replacement Timing}

Until now we analyzed the impact of uncertainty and strategic interactions on the optimal replacement threshold of the firm. Although threshold values and timing have a lot to do with each other, it cannot be concluded in general that the relation between the two is monotonic (cf. Sarkar, 2000). After having determined the dependency of threshold values on uncertainty in Section 5 and 6, in this section we investigate the relationship between uncertainty, expected timing of replacement and the probability with which the threshold is reached within a time interval of a given length.

First, let us observe that the expectation of the first passage time equals ${ }^{19}$

$$
E\left[T^{*}\right]=\frac{1}{\alpha-\frac{1}{2} \sigma^{2}} \ln \frac{A^{*}\left(\sigma^{2}\right)}{A},
$$

where $A^{*}\left(\sigma^{2}\right)$ denotes the optimal replacement threshold as a function of uncertainty. We note that expectation (49) tends to infinity for $\sigma^{2} \rightarrow 2 \alpha$ and does not exist for $\sigma^{2}>2 \alpha{ }^{20}$ For $\sigma^{2}<2 \alpha$ it holds that

$$
\frac{\partial E\left[T^{*}\right]}{\partial\left(\sigma^{2}\right)}=\frac{1}{2\left(\alpha-\frac{1}{2} \sigma^{2}\right)^{2}} \ln \frac{A^{*}\left(\sigma^{2}\right)}{A}+\frac{1}{\alpha-\frac{1}{2} \sigma^{2}} \frac{\frac{d A^{*}}{d\left(\sigma^{2}\right)}}{A^{*}\left(\sigma^{2}\right)}>0 .
$$

The expected timing of replacement increases in uncertainty due to two effects. First, for any given threshold, the associated expected first passage time is increasing in uncertainty (cf. the first component of the RHS of (50)). Second, for a fixed level of uncertainty, an increase in the optimal investment threshold leads to an increase in the expected time to reach (cf. second component of RHS of (50)). Based on (50) it can be concluded that whenever the threshold goes up due to more uncertainty, it also holds that the expected time to replace the production facility increases.

An alternative approach to measure the impact of uncertainty on the timing of replacement is to look at the probability with which the threshold is reached within a time interval of a given length, say $\tau$. Contrary to the expected first passage time, this approach does not impose any restrictions on the values of $\sigma$. The probabilities of optimal asset replacement within a given interval are particularly useful when this interval coincides with a budgeting period. ${ }^{21}$

\footnotetext{
${ }^{19}$ For a derivation of the probability distribution of the first passage time see Harrison (1985) for a formal exposition and Dixit (1993) for a heuristic approach.

${ }^{20}$ Increasing $\sigma^{2}$ beyond $2 \alpha$ implies that the probabilities of surviving without reaching the threshold before a given time do not fall sufficiently fast for longer hitting times (moreover, the probability that the process will reach the barrier in infinity is still positive). Since the expectation is the sum of the product of the first passage times and their probabilities, an insufficient decay in the survival probabilities (without reaching the threshold) results in the divergence of the expectation.

${ }^{21}$ For a discussion of the capital budgeting process at the corporate level see Kaplan and Atkinson (1998), Ch. 14 and Bower (1986), Ch. 1-3.
} 
The formula for the probability of hitting the optimal replacement threshold before time $\tau$ by a geometric Brownian motion can be derived using, for instance, Harrison (1985, Equation 8.11). Consequently, it holds that

$$
\begin{aligned}
P(T<\tau)= & \Phi\left(\frac{-\ln \frac{A^{*}}{A}+\left(\alpha-\frac{1}{2} \sigma^{2}\right) \tau}{\sigma \sqrt{\tau}}\right) \\
& +\left(\frac{A^{*}}{A}\right)^{\frac{2 \alpha}{\sigma^{2}}-1} \Phi\left(\frac{-\ln \frac{A^{*}}{A}-\left(\alpha-\frac{1}{2} \sigma^{2}\right) \tau}{\sigma \sqrt{\tau}}\right),
\end{aligned}
$$

where $T$ denotes the time to reach the threshold and $\Phi$ is the standard normal cumulative density function. As already pointed out in Sarkar (2000), the derivative $\frac{\partial P(T<\tau)}{\partial \sigma}$ does not have an unambiguous sign and it can thus be shown that, in general, uncertainty can affect the probability of reaching the threshold within a given time in both directions.

First, we illustrate the relationship between the first passage time, volatility and related probabilities for the follower threshold since this threshold is unaffected by strategic considerations. Subsequently, we present results of simulations related to the threshold of the leader. In this part we use the model of Sections 3-4. The results for the decision to start production are qualitatively similar and are not reported. ${ }^{22}$

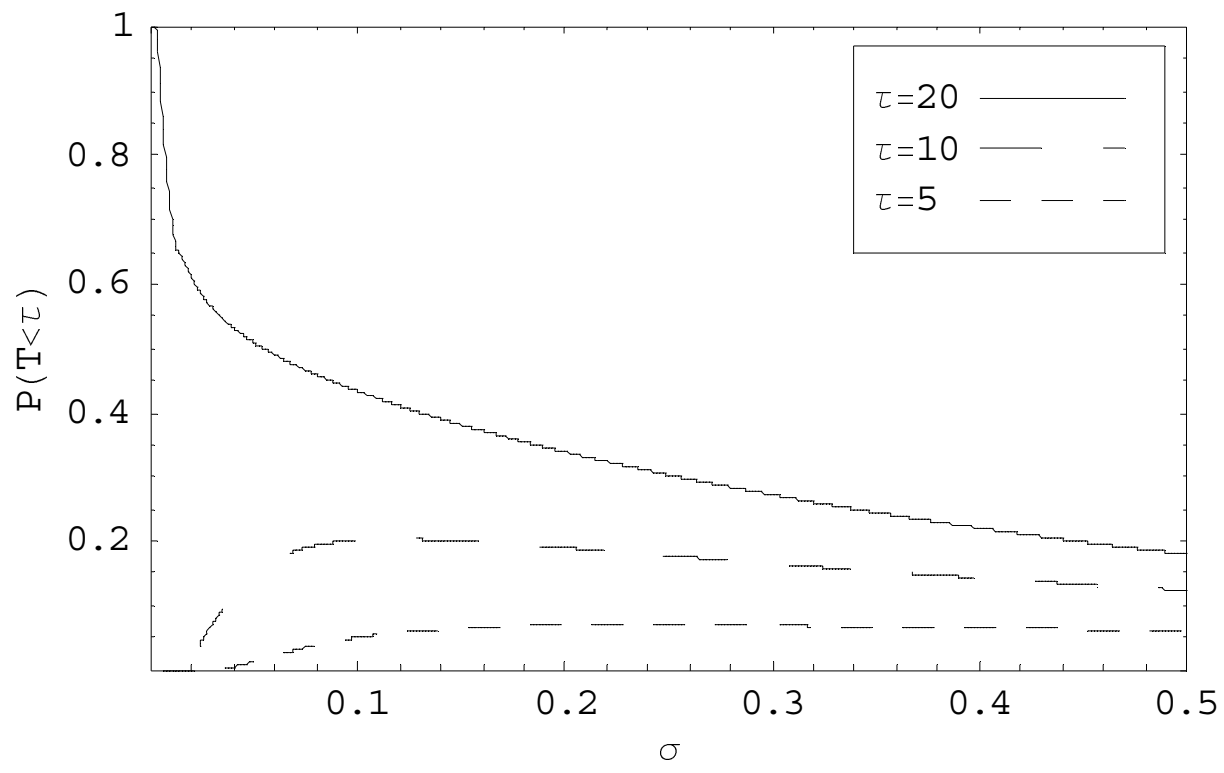

Figure 3: The cumulative probability of reaching the optimal follower replacement threshold as a function of demand uncertainty for a set of parameter values: $A=4, r=0.05, \alpha=0.015, K=3$, $k=0$ and $I=60$.

From Figure 3 it can be concluded that the form of the relationship between the

\footnotetext{
${ }^{22}$ In this case a restriction on $\sigma$ has to be imposed in order to ensure a positive sign of $\varrho$ (cf. (12)).
} 
uncertainty and the probability of reaching the threshold depends on the length of the time interval. For sufficiently long time intervals, the cumulative probability of reaching the threshold decreases in volatility. Intuitively, this can be explained by the fact that the probability mass of the first passage time density function moves to the right (cf. (50)) and longer times of reaching the demand level triggering replacement become more likely. Moreover, the trigger itself is increasing in $\sigma$.

For low values of $\tau$ the probability of reaching the replacement threshold first increases and then decreases. For $\sigma=0$ the probability of reaching the threshold within a certain time interval is zero when the optimal replacement time lies outside this interval. Increasing $\sigma$ results in a spread of the probability mass, so that the probability of reaching the demand threshold becomes positive for a strictly positive $\sigma$. A larger spread is initially equivalent to a higher probability of hitting the optimal replacement threshold. However, when volatility continues to rise, at a certain moment the effect of the probability mass shifting to the right starts to dominate the effect of the spread. As a consequence, the cumulative probability of reaching the threshold becomes smaller again.

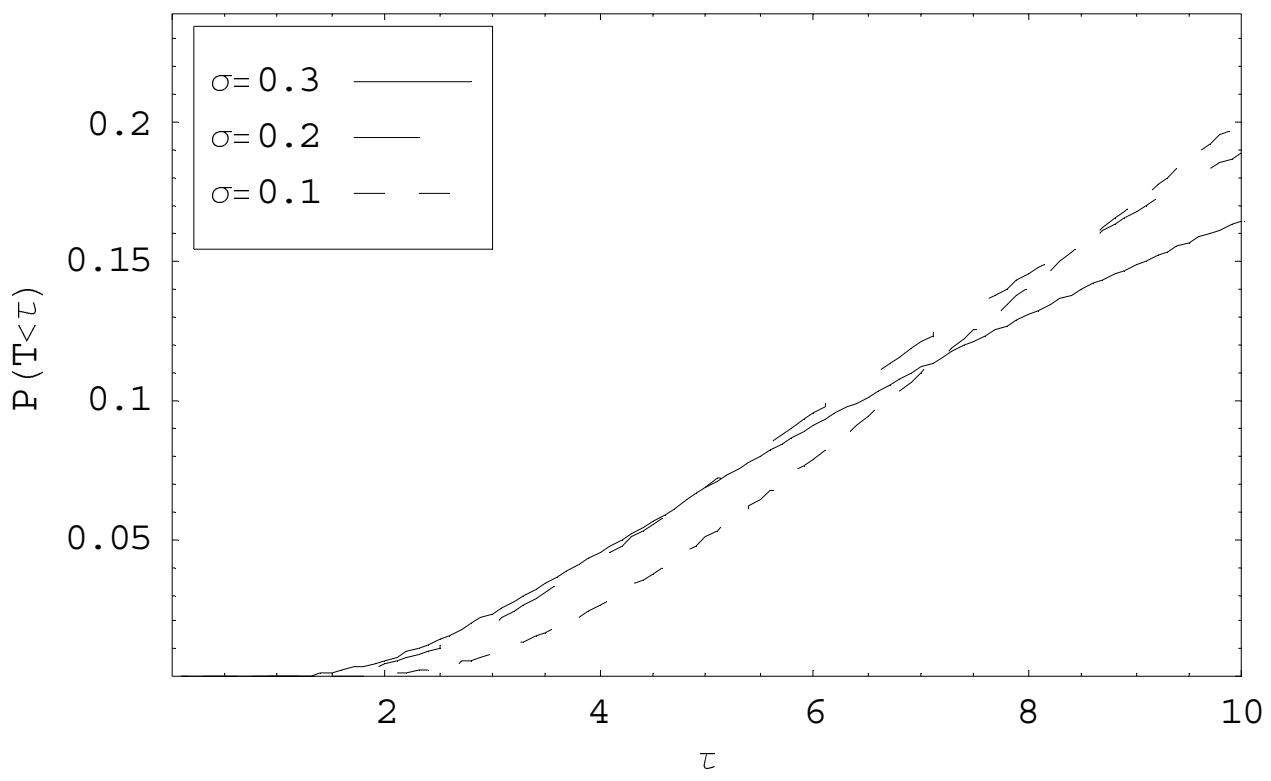

Figure 4: The cumulative probability of reaching the optimal follower replacement threshold as a function of time horizon for the set of parameter values: $A=4, r=0.05, \alpha=0.015, K=3$, $k=0$ and $I=60$.

Figure 4 indicates that the probability of reaching the follower threshold always increases with the time interval, which is of course trivial. The relevant observation is that this relationship is more pronounced for low levels of market uncertainty. This results from the fact that in the absence of uncertainty the optimal investment trigger is reached at a 
specified point in time with probability 1 and the corresponding cumulative density function is a heaviside step function. Increasing volatility spreads the probability mass around the point corresponding to the deterministic case. This leads to an increased cumulative chance of reaching the trigger at points in time situated to the left of this specified point in time, while the reverse is true for the point situated to the right. This influences the shape of the cumulative distribution function whose slope decreases in uncertainty.

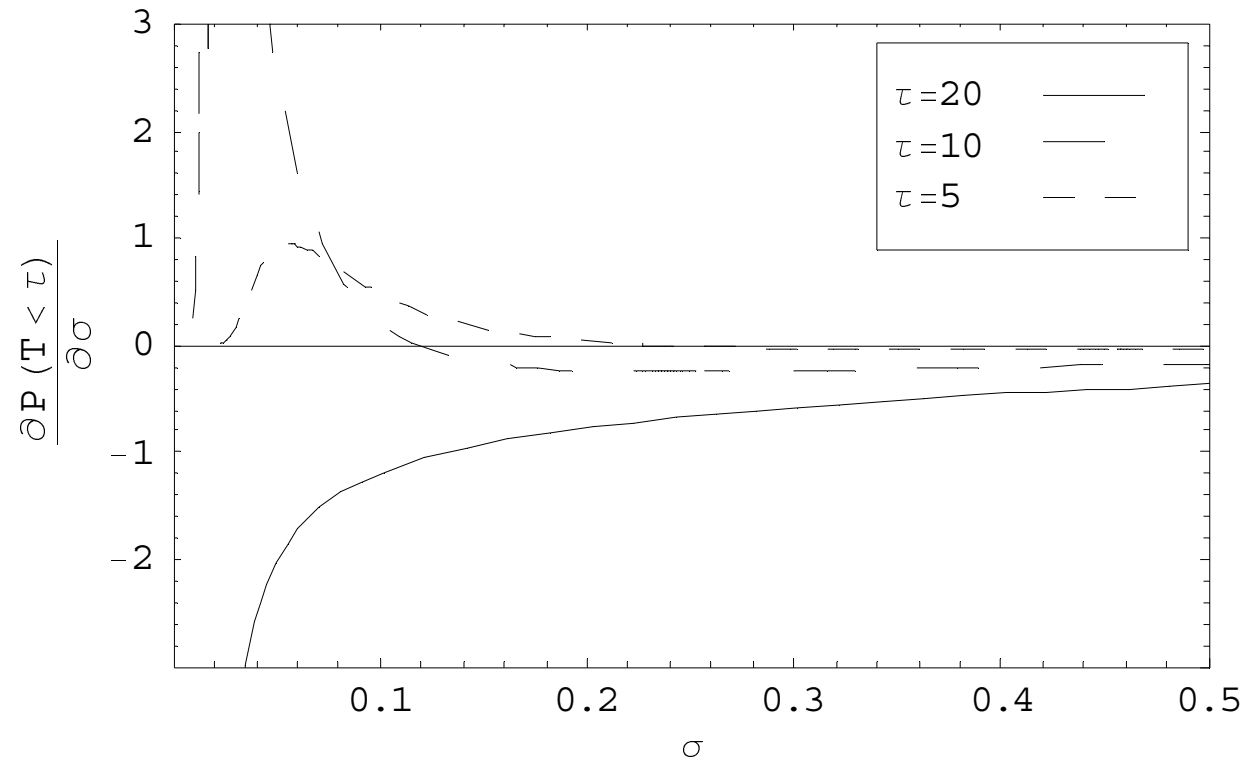

Figure 5: The derivative with respect to market uncertainty of the cumulative probability of reaching the optimal follower threshold as a function of uncertainty for the set of parameter values: $A=4$, $r=0.05, \alpha=0.015, K=3, k=0$ and $I=60$.

Figure 5 allows for a closer inspection of the relationship between the timing of asset replacement and uncertainty. It can be concluded that, irrespective from the length of the time interval, there exists a level of uncertainty beyond which a further increase in uncertainty always reduces the probability of the optimal asset replacement. The relationship between this level and the length of the time interval is inverse, i.e. the longer the time interval, the lower level of uncertainty for which a further uncertainty increase reduces the probability of the optimal replacement. For example, using the parameters from Figure 5 we can conclude that for $\tau=5$ this critical value of uncertainty, $\sigma$, is 0.234 , for $\tau=10$ it is only 0.118 , whereas for $\tau=20$ increased uncertainty always reduces the cumulative probability of optimal investment.

Figure 6 indicates that the probability of the optimal replacement increases in uncertainty for a sufficiently short time interval and decreases for a sufficiently long horizon. Moreover, the derivative of the probability of reaching the optimal threshold changes its sign 


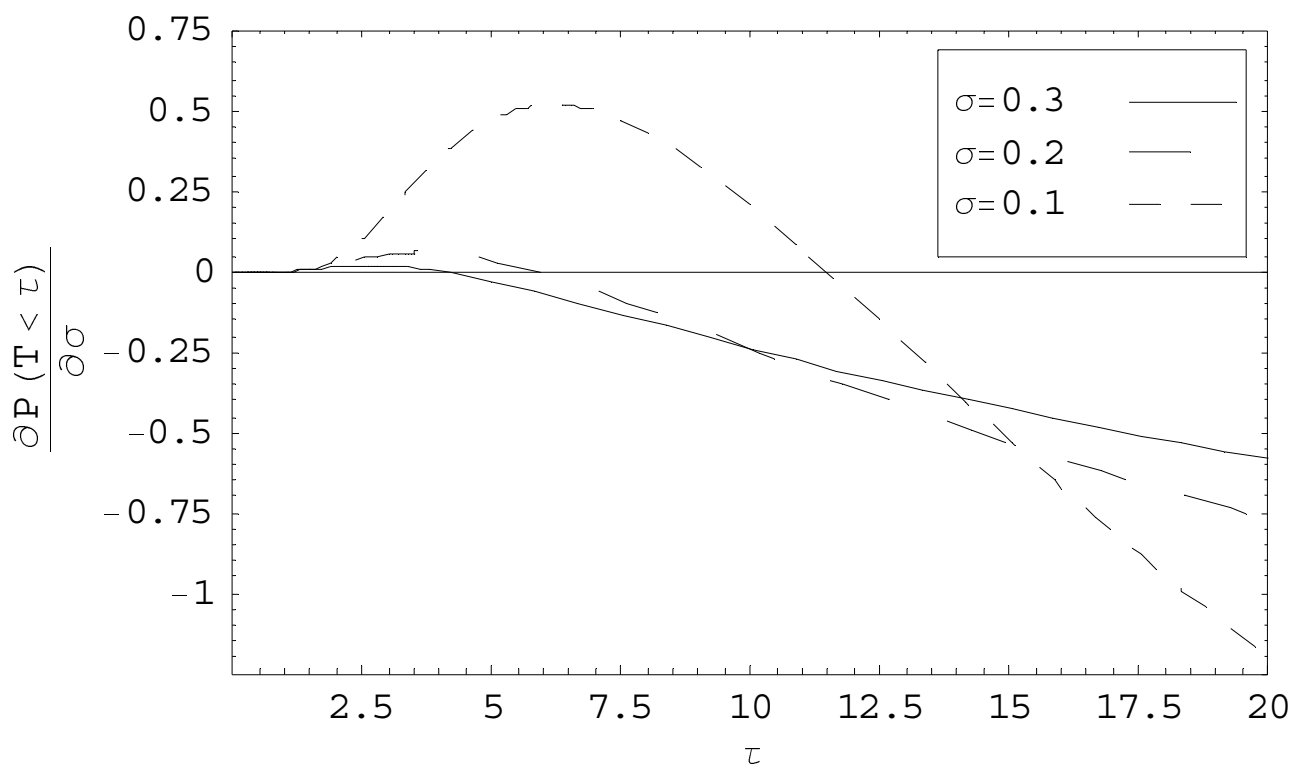

Figure 6: The derivative with respect to market uncertainty of the cumulative probability of reaching the optimal follower threshold as a function of time horizon for the set of parameter values: $A=4$, $r=0.05, \alpha=0.015, K=3, k=0$ and $I=60$.

only once. Finally, Figure 6 allows for the conclusion that the length of time interval beyond which uncertainty negatively affects the probability of optimal replacement is, again, negatively related to the uncertainty level. For $\sigma=0.1$ the interval length which separates the areas of a positive and a negative relationship equals 11.46 years, for $\sigma=0.2$ it equals 5.87 years, while for $\sigma=0.3$ it drops to 4.06 years.

Despite the presence of strategic effects, the probability of asset replacement of the leader within a given time interval responds to changes in uncertainty and the length of the interval in a similar way as the corresponding probabilities of the follower. For low $\sigma$ 's the probability of investing increases more rapidly with the length of the time interval than for high $\sigma$ 's. Moreover, for high $\tau$ 's the probability of replacing the existing asset is always decreasing in uncertainty, while for low $\tau$ 's the probability behaves in a non-monotonic way.

The results of the simulations concerning the relationship between uncertainty, first passage time and probabilities of reaching the leader threshold are presented in Table 1 below. 


\begin{tabular}{l|rrrrrr}
\hline \hline$\sigma$ & $\tau=1$ & $\tau=2$ & $\tau=5$ & $\tau=10$ & $\tau=15$ & $\tau=20$ \\
\hline 0.05 & 0.06 & 2.39 & 24.11 & 54.32 & 71.17 & 80.97 \\
0.10 & 0.61 & 5.93 & 26.79 & 47.94 & 59.70 & 67.24 \\
0.20 & 0.62 & 5.14 & 21.00 & 36.47 & 45.10 & 50.72 \\
0.30 & 0.46 & 3.97 & 16.50 & 28.66 & 35.31 & 39.55 \\
0.40 & 0.39 & 3.30 & 13.57 & 23.22 & 28.29 & 31.39 \\
0.50 & 0.36 & 2.93 & 11.55 & 19.23 & 23.02 & 25.21 \\
\hline \hline
\end{tabular}

Table 1: The cumulative probability (in percentages) of reaching the optimal leader replacement threshold as a function of demand uncertainty for the set parameter values: $A=2, r=0.05, \alpha=$ $0.015, k=0, K=3$ and $I=60$.

The relationship between the investment probability of the leader and uncertainty is analogous to the corresponding relationship of the follower. The probability that the leader replaces its production facility within a given time interval decreases with uncertainty when the length of this interval is sufficiently large. In a situation where the relevant interval is sufficiently short, there are two contradictory effects. On the one hand, the investment probability increases because higher volatility enhances the chance of reaching a particular threshold early. On the other hand, this probability eventually declines with uncertainty because then the effect of the probability mass shifting to the right begins to dominate.

Now, we formulate the following proposition, which extends Sarkar (2000) by defining the time interval lengths separating a monotonic and non-monotonic relationship between uncertainty and the investment probability .

\section{Proposition 4 Define}

$$
\tau^{*} \equiv \frac{1}{\alpha} \ln \frac{A^{*}}{A}, \alpha>0,
$$

as the point in time at which the replacement threshold $A^{*}$ is reached in the deterministic case. Then it holds that for $\tau<\tau^{*}$ the probability of reaching the investment threshold $A^{*}$ before $\tau$ increases in uncertainty at a relatively low level of uncertainty and decreases for a relatively high level, whereas for $\tau>\tau^{*}$ the probability of reaching the optimal threshold before $\tau$ always decreases in uncertainty.

Proof. See Appendix A.

On the basis of Proposition 4 it may be concluded that the replacement horizon being equal to the optimal timing of replacement in the deterministic case separates the regions of monotonic and non-monotonic relationship between uncertainty and the probability of replacement. In Table 1, the parameters are chosen in such a way that the optimal timing of replacement in the deterministic case equals $\tau^{*}=9.36$. Therefore the investment-uncertainty relationship in columns $2-4$ is non-monotonic, while it is negative in columns $5-7$. 
In order to determine $\tau^{*}$ for the leader, we need to determine its replacement timing in the deterministic case. It holds that the optimal investment timing of the leader in the model without uncertainty is equal to the rent equalization point in the preemption game of Fudenberg and Tirole (1985). In the case of the follower, $\tau^{*}$ is equal to the point of time at which the incremental flow from operations, $\pi^{11}-\pi^{01}$, reaches the flow associated with the replacement cost, $I r$. Hence, $\tau^{*}$ corresponds to the optimal Jorgensonian trigger, which equalizes the flow costs and revenues of the project. The optimal simultaneous replacement closely resembles the case of the follower trigger. The only difference is that now the incremental profit flow equals $\pi^{11}-\pi^{00}$.

Finally, we would like to point out that our analysis also extends to the situation where $\alpha \leq 0$. In such a case and without uncertainty the firms would face now-or-never decisions. Therefore, it holds that $\tau^{*} \in\{0, \infty\}$, so that the relationship between the investment probability within a given time interval and uncertainty will be either non-monotonic or decreasing for all time horizons. This implies that in mature industries (i.e. those with non-positive growth rate), the probability of launching existing positive NPV projects always decreases with uncertainty. As far as initially negative NPV projects are concerned, the probability of their optimal execution is initially increasing in uncertainty. When uncertainty becomes sufficiently high, the replacement probability starts to fall.

\section{Conclusions}

The purpose of this paper is to analyze the firm's decision to replace an existing production facility with a technologically superior one. In order to capture the effect of strategic interactions among the firms operating in an imperfectly competitive and uncertain environment we model the product market as a Cournot duopoly with a stochastic demand parameter. Such a formulation results in the payoff functions being convex in the stochastic demand parameter.

We determine the types of equilibria of the real option game played by the firms. We show that it is optimal for the firms to replace their production facilities sequentially when the associated cost is relatively low and simultaneously otherwise.

Furthermore, we find that the direct effect of uncertainty (related to the waiting option) on the replacement threshold of the leader is always larger than the indirect effect (strategic option) resulting from the delay in the follower decision to replace its production facility. Consequently, irrespective from the type of equilibrium, increasing uncertainty always raises the level of demand triggering the optimal replacement. This result also holds in case of the decision to start production rather than to replace the existing asset.

Moreover, it can be concluded that the expected timing of replacement increases with uncertainty. This result supports the view that uncertainty delays the implementation of the 
new technology, even in the presence of strategic interactions combined with a convex profit function. Moreover, it shows that the result of Kulatilaka and Perotti (1998) that uncertainty can stimulate investment due to strategic interactions does not carry over from a two period model to a continuous-time setting.

We also determine the probability of replacing the production facility within a certain time interval. Here, the point in time at which replacement is made optimally in the deterministic case plays a crucial role. For an interval that contains this point in time, the probability of optimal replacement within this time interval decreases with uncertainty. However, if this time interval is that short that the optimal replacement time in the deterministic case lies outside this interval, then the replacement probability goes up with uncertainty when uncertainty is low while it goes down otherwise.

Finally, we would like to discuss the limitations of our approach. In order to ensure analytical tractability and to make our results comparable to Kulatilaka and Perotti (1998), we used a linear demand specification and parallel shifts in demand. Such a model specification allowed us to show that in a continuous-time framework the convexity of payoff functions does not result in investment occurring at lower states of demand when uncertainty is higher, as it does in a two-period model. Our setting enabled us to show that uncertainty enhancing convex payoffs and therefore stimulating investment in a two-period case does not accelerate strategic replacement when there is flexibility in timing the replacement. Of course, our predictions do not automatically carry over to other forms of product market uncertainty. In other words, we do not formally define the classes of demand functions for which the analysis holds. With respect to robustness of our results, it is also important to relax other assumptions like constant marginal costs and Cournot competition. We plan to pursue these issues in future work. 


\section{A Proofs of Propositions}

Proof of Proposition 1. First, let us define

$$
\zeta(A) \equiv V^{S}(A)-V^{L}(A) .
$$

Here, we assume $k=0$. After substituting (20) and (24) into (A.1) we get ${ }^{23}$

$$
\zeta(A)=-\frac{4 K A}{9 \delta}+I+\left(\frac{\frac{1}{\beta-1}\left(I+\frac{K^{2}}{9 r}\right)}{\left(A^{S}\right)^{\beta}}+\frac{\frac{\beta}{2(\beta-1)}\left(I+\frac{4 K^{2}}{9 r}\right)+\frac{K^{2}}{9 r}}{\left(A^{F}\right)^{\beta}}\right) A^{\beta}
$$

for $A \leq A^{F}$. From (33) it follows that if on the interval $\left[A^{P}, A^{F}\right]$ the minimum of $\zeta(A)$ is smaller than zero, a preemptive equilibrium occurs. Otherwise, the firms replace their production facilities simultaneously. ${ }^{24}$ The existence of a negative minimum of $\zeta(A)$ depends on the value of input parameters. The minimum of $\zeta(A)$ occurs for

$$
A^{* *}=\left(\frac{\frac{4 K}{9 \beta \delta}\left(A^{S} A^{F}\right)^{\beta}}{\frac{1}{\beta-1}\left(I+\frac{K^{2}}{9 r}\right)\left(A^{F}\right)^{\beta}+\left(\frac{\beta}{2(\beta-1)}\left(I+\frac{4 K^{2}}{9 r}\right)+\frac{K^{2}}{9 r}\right)\left(A^{S}\right)^{\beta}}\right)^{\frac{1}{\beta-1}} .
$$

The type of equilibrium is uniquely determined by the sign of $\zeta\left(A^{* *}\right)$. First, we show that $\zeta\left(A^{* *}\right)$ is increasing in $I$. Then, to prove the existence of a unique value of $I$ for which $\zeta\left(A^{* *}\right)=0$, we establish that $\zeta\left(A^{* *}\right)$ is negative for a sufficiently small $I$ and that it is positive for $I$ tending to infinity.

Consequently, we first show that $\zeta\left(A^{* *}\right)$ is increasing with $I$, i.e. that the following inequality holds

$$
\left.\frac{d \zeta(A)}{d I}\right|_{A=A^{* *}}=\left.\frac{\partial \zeta(A)}{\partial I}\right|_{A=A^{* *}}+\left.\frac{\partial \zeta(A)}{\partial A}\right|_{A=A^{* *}} \frac{d A^{* *}}{d I}>0 .
$$

Using the fact that

$$
\left.\frac{\partial \zeta(A)}{\partial A}\right|_{A=A^{* *}}=0
$$

and differentiating (A.2), we can express $\frac{d \zeta(A)}{d I}$ as

$$
\frac{d \zeta(A)}{d I}=1-\left(\frac{A}{A^{S}}\right)^{\beta}-\beta \frac{\frac{I}{2}+\frac{K^{2}}{3 r}}{I+\frac{4 K^{2}}{9 r}}\left(\frac{A}{A^{F}}\right)^{\beta} .
$$

Subsequently, we substitute for $A$ in (A.6) the expression (A.3) for $A^{* *}$. Complexity of the resulting expression yields the necessity to use a numerical procedure. A geometric grid

\footnotetext{
${ }^{23}$ The proof for general $k$ goes along the same lines and is skipped for the sake of brevity.

${ }^{24}$ Strictly speaking, the preemptive equilibrium still exists in this case but is Pareto-dominated by the simultaneous replacement equilibrium (cf. Fudenberg and Tirole, 1985).
} 
search indicates that $\left.\frac{d \zeta\left(A_{t}\right)}{d I}\right|_{A=A^{* *}}$ is positive for $\beta \in\left[1, \frac{r}{\alpha}\right)$, and $\alpha \in(0, \infty), \beta \in[1, \infty)$, and $\alpha \in(-\infty, 0]$, and for other parameters falling into intervals: $r \in(\alpha, \infty), K \in(0, \infty)$ and $I \in\left(\frac{4 K^{2}}{9 r}, \infty\right) \cdot{ }^{25}$

To conclude that $\zeta\left(A^{* *}\right)$ is negative for a sufficiently small $I$, we use the following properties of $\zeta(A): i) \zeta\left(A^{* *}\right)$ is bounded from above by $\zeta\left(A^{L}\right)$, where $A^{L}$ is given by $\frac{\beta}{\beta-1} \frac{9 I \delta}{4 K}$, and $i$ i) $\zeta\left(A^{L}\right)$ is negative for the smallest economically relevant value of replacement cost $I=\frac{4 K^{2}}{9 r}$ (cf. footnote 22). Property $i$ ) follows from the definition of $A^{* *}$. Property $\left.i i\right)$ can be established by observing that $\zeta\left(A^{L} ; \frac{4}{9} \frac{K^{2}}{r}\right)=\frac{4 K^{2}}{9 r} \frac{1}{\beta-1}\left[-1+\frac{1}{2}\left(\frac{2}{5}\right)^{\beta-1}+(5 \beta-1)\left(\frac{1}{2}\right)^{\beta+2}\right]$, and that the expression between brackets is negative for $\beta \in(1, \infty)$.

Finally, a positive sign of $\lim _{I \rightarrow \infty} \zeta\left(A^{* *}\right)=I\left[1-\left(\left(\frac{1}{2}\right)^{\beta}+\frac{1}{2} \beta\right)\right]^{\frac{1}{1-\beta}}$ can be established by observing that the expression between brackets is positive for $\beta \in(1, \infty)$.

Proof of Proposition 2. Differentiating (25) (for $A \leq A^{F}$ ) yields

$$
\frac{d \xi(A)}{d \beta}=\frac{\left(\frac{\beta}{\beta-1}\left(\frac{3 I}{2}+\frac{2 K^{2}}{3 r}\right)-\frac{K^{2}}{3 r}-I\right) \ln \left(\frac{A^{F}}{A}\right)-\frac{1}{\beta-1}\left(\frac{I}{2}+\frac{K^{2}}{3 r}\right)}{\left(\frac{A^{F}}{A}\right)^{\beta}} .
$$

Since the threshold of the leader is equal to $A^{P}$, and $A^{P}$ is the smallest root of the concave function $\xi(A)$, we know that

$$
\left.\frac{\partial \xi(A)}{\partial A}\right|_{A=A^{P}}>0
$$

Consequently, by differentiating (25) totally, we conclude that it is sufficient to show that

$$
\left.\frac{d \xi(A)}{d \beta}\right|_{A=A^{P}}>0
$$

to conclude that the replacement threshold of the leader is increasing with uncertainty (decreasing with $\beta$ ). Moreover, upon analyzing (A.7) we know that $\frac{d \xi(A)}{d \beta}$ changes its sign only once and the corresponding realization of $A$ to the zero value of the derivative is

$$
A^{*}=A^{F} e^{-\frac{\frac{I}{2}+\frac{K^{2}}{3 r}}{\beta\left(\frac{I}{2}+\frac{K^{2}}{3 r}\right)+\frac{K^{2}}{3 r}+I}} .
$$

Therefore

$$
\frac{d \xi(A)}{d \beta}>0 \text { iff } A<A^{*}
$$

Consequently, $\xi\left(A^{*}\right)>0$ would imply that $A^{*}>A^{P}$ and $\left.\frac{d \xi(A)}{d \beta}\right|_{A=A^{P}}>0$. In order to prove

\footnotetext{
${ }^{25}$ The domain of $I$ results from the fact that for this range of values of $K$, thresholds $A^{F}$ and $A^{S}$ are decreasing in $K$, which constitutes the economically relevant case.
} 
that $\xi\left(A^{*}\right)>0$, we plug (A.10) into (25) to obtain

$$
\begin{aligned}
\xi\left(A^{*}\right)= & \frac{\beta}{\beta-1}\left(\frac{3 I}{2}+\frac{2 K^{2}}{3 r}\right) e^{-\frac{\frac{I}{2}+\frac{K^{2}}{3 r}}{\beta\left(\frac{I}{2}+\frac{K^{2}}{3 r}\right)+\frac{K^{2}}{3 r}+I}}-\frac{K^{2}}{3 r}-I \\
& -\left(\frac{\beta}{\beta-1}\left(\frac{3 I}{2}+\frac{2 K^{2}}{3 r}\right)-\frac{K^{2}}{3 r}-I\right) e^{-\beta \frac{\frac{I}{2}+\frac{K^{2}}{3 r}}{\beta\left(\frac{I}{2}+\frac{K^{2}}{3 r}\right)+\frac{K^{2}}{3 r}+I}} .
\end{aligned}
$$

An analytical proof is again not possible but numerically it can be shown that $\xi\left(A^{*}\right)$ is positive for $\beta \in\left[1, \frac{r}{\alpha}\right)$, and $\alpha \in(0, \infty), \beta \in[1, \infty)$, and $\alpha \in(-\infty, 0]$, and for other parameters falling into intervals: $r \in(\alpha, \infty), K \in(0, \infty)$ and $I \in\left(\frac{4 K^{2}}{9 r}, \infty\right)$.

Proof of Proposition 3. We rewrite the derivative (47) as

$$
\begin{aligned}
& \frac{d \xi^{N}(A)}{d\left(\sigma^{2}\right)}=\frac{A^{2}}{4 \varrho^{2}}-\frac{5 I}{4(\beta-2)}\left(\frac{A}{A^{F N}}\right)^{\beta} \\
& \times\left[\frac{\partial \beta}{\partial\left(\sigma^{2}\right)}+\left(\beta+\frac{8}{5}\right)\left(\frac{\beta}{2 \varrho}+\ln \left(\frac{A}{A^{F N}}\right) \frac{\partial \beta}{\partial\left(\sigma^{2}\right)}\right)\right] .
\end{aligned}
$$

Denote the smallest solution of $\xi^{N}(A)=0$ by $A^{P N}$. Since $A^{P N}$ cannot be explicitly derived, we proceed as follows. First, we consider a particular point $\bar{A}>A^{P N}$. Second, we show that $\frac{d \xi^{N}(A)}{d\left(\sigma^{2}\right)}$ is negative for all $A \in(\underline{A}, \bar{A})$, where $\underline{A}$ is a realization of $A$ such that $\underline{A}<A^{P N}$. Let us define

$$
\bar{A} \equiv 2 \sqrt{\frac{\beta}{\beta-2} I \varrho}
$$

First, we show that $\xi^{N}(\bar{A})>0$, which would imply that $\bar{A}>A^{P N}$. After substituting (A.14) into (46) we obtain that

$$
\xi^{N}(\bar{A}, \beta)=\frac{2 I}{\beta-2}\left(1-\left(\frac{5}{8} \beta+1\right)\left(\frac{2}{3}\right)^{\beta}\right)=\frac{2 I}{\beta-2} \phi(\beta) .
$$

Since $\beta>2$ (recall that for $\beta \leq 2$ no firm is willing to enter), we know that $\frac{2 I}{\beta-2}$ is always positive. Therefore we are interested only in the sign of $\phi(\beta)$. For $\beta \downarrow 2$ we obtain that

$$
\lim _{\beta \downarrow 2} \phi(\beta)=0
$$

Then we establish that

$$
\frac{\partial \phi(\beta)}{\partial \beta}=-\left(\frac{2}{3}\right)^{\beta}\left(\frac{5}{8}+\left(\frac{5}{8} \beta+1\right) \ln \left(\frac{2}{3}\right)\right)>0
$$

for $\beta \in(2, \infty)$. This implies that $\xi^{N}(\bar{A})$ is positive so that $\bar{A}>A^{P N}$. We proceed with proving that expression (A.13) changes signs twice, i.e. it is positive for $A \in(0, \underline{A}) \cup\left(\overline{\bar{A}}, A^{F N}\right)$, 
where $\overline{\bar{A}}$ is some realization of $A$ such that $\overline{\bar{A}}>\bar{A}$, and negative otherwise. First, we express (A.13) as

$$
\frac{d \xi^{N}(A)}{d\left(\sigma^{2}\right)}=A^{2}\left[K A^{\beta-2}+L A^{\beta-2} \ln \left(\frac{A}{A^{F N}}\right)+M\right]
$$

where

$$
\begin{aligned}
K & =-\frac{5 I}{4(\beta-2)}\left(A^{F N}\right)^{-\beta}\left(\frac{\partial \beta}{\partial\left(\sigma^{2}\right)}+\frac{\beta\left(\beta+\frac{8}{5}\right)}{2 \varrho}\right), \\
L & =-\frac{5 I}{4(\beta-2)}\left(A^{F N}\right)^{-\beta}\left(\beta+\frac{8}{5}\right) \frac{\partial \beta}{\partial\left(\sigma^{2}\right)}>0, \text { and } \\
M & =\frac{1}{4 \varrho^{2}}>0 .
\end{aligned}
$$

From (A.18)-(A.21) it can be derived that ${ }^{26}$

$$
\begin{aligned}
\lim _{A \downarrow 0} K A^{\beta-2}+L A^{\beta-2} \ln \left(\frac{A}{A^{F N}}\right)+M & =M, \text { and } \\
\lim _{A \rightarrow \infty} K A^{\beta-2}+L A^{\beta-2} \ln \left(\frac{A}{A^{F N}}\right)+M & =\infty .
\end{aligned}
$$

Moreover

$$
\begin{aligned}
& \frac{\partial}{\partial A}\left(K A^{\beta-2}+L A^{\beta-2} \ln \left(\frac{A}{A^{F N}}\right)+M\right) \\
= & A^{\beta-3}\left((\beta-2) K+(\beta-2) L \ln \left(\frac{A}{A^{F N}}\right)+L\right),
\end{aligned}
$$

which implies that there exists only one extremum of $\frac{d \xi^{N}(A)}{d\left(\sigma^{2}\right)}$ that is different from zero. This result, combined with (A.22) and (A.23), implies that $\frac{d \xi^{N}(A)}{d\left(\sigma^{2}\right)}$ is negative at most in only one interval. Substituting $\bar{A}$ into (A.13) yields

$$
\begin{aligned}
& \left.\frac{d \xi^{N}(A)}{d\left(\sigma^{2}\right)}\right|_{A=\bar{A}}=\frac{2 I}{(\beta-2) \varrho}-\frac{5 I}{4(\beta-2)}\left(\frac{2}{3}\right)^{\beta} \\
& \times\left[\frac{\partial \beta}{\partial\left(\sigma^{2}\right)}+\left(\beta+\frac{8}{5}\right)\left(\frac{\beta}{2 \varrho}+\ln \left(\frac{2}{3}\right) \frac{\partial \beta}{\partial\left(\sigma^{2}\right)}\right)\right]
\end{aligned}
$$

Numerically it can be shown that $\left.\frac{\partial \xi^{N}(A)}{\partial\left(\sigma^{2}\right)}\right|_{A=\bar{A}}$ is negative for $\beta \in\left[1, \frac{r}{\alpha}\right)$, and $\alpha \in(0, \infty)$, $\beta \in[1, \infty)$, and $\alpha \in(-\infty, 0], \alpha \in \mathbb{R}, r \in(\alpha, \infty)$ and $I \in(0, \infty)$. Therefore the only remaining part of the proof is to show that $\underline{A}<A^{P N}$ for any vector of input parameters. Since the explicit analytical forms of $\underline{A}$ and $A^{P N}$ do not exist, we use a numerical procedure. Using a grid search technique (for the domains of input parameters as in the proofs of Propositions 3.1 and 3.2 ), we calculate the differences $A^{P N}-\underline{A}$ and it turns out that they are always

\footnotetext{
${ }^{26}$ The result (A.22) has been derived using l'Hôpital's rule.
} 
positive. Given that $\left.\frac{d \xi^{N}(A)}{d\left(\sigma^{2}\right)}\right|_{A \in(\underline{A}, \bar{A}]}<0, A^{P N} \in(\underline{A}, \bar{A})$ and $\xi^{N}(\bar{A})>0$, we conclude that $\frac{d A^{P N}}{d\left(\sigma^{2}\right)}>0$, i.e. the investment threshold of the leader increases with uncertainty.

Proof of Proposition 4. First, we show that $\tau^{*}$ is the time to reach the replacement threshold $A^{*}$ in the deterministic case. After observing that $x=\alpha t$ is the solution to $d x=\alpha d t$ with initial condition $x(0)=0$, and substituting $x^{*}=\ln \frac{A^{*}}{A}$, we obtain that

$$
\ln \frac{A^{*}}{A}=\alpha \tau^{*}
$$

so $\tau^{*}$ in (52) is the time to reach the threshold $A^{*}$. Now, we consider the density function $\varphi\left(\tau ; \mu(\sigma), \sigma^{2}\right)$ being the density function of the first passage time for a geometric Brownian motion, which has a mean $\mu(\sigma)$ and variance $\sigma^{2}$. For the moment we assume that $\mu=$ $\tau^{*}$ irrespective from $\sigma$. Then, raising the variance $\sigma^{2}$ is equivalent to performing a mean preserving spread. Consequently, in such a case

$$
\frac{\partial}{\partial \sigma}\left(\int_{0}^{\tau} \varphi(s) d s\right)\left(\tau-\tau^{*}\right) \leq 0
$$

with equality holding if and only if $\tau=\tau^{*}$. The expectation of the first passage time, $E[\tau]$, associated with hitting the replacement threshold $A^{*}$, is increasing with $\sigma$ (cf. (50)) and $A^{*}$ is increasing with $\sigma$, too. For $\tau>\tau^{*}$, an increase in uncertainty not only reduces the probability mass to the left of $\tau$ via the mean preserving spread but also because of the mean itself moving to the right. Therefore the effect of uncertainty on the probability of the replacement decision is unambiguous in this region and negative. For $\sigma \rightarrow \infty$ the probability of investing before $\tau$ decreases to zero. The latter conclusion is true since from (51) it is obtained that

$$
\begin{aligned}
\lim _{\sigma \rightarrow \infty} P(T<\tau)= & \lim _{\sigma \rightarrow \infty} \Phi\left(\frac{-\ln \frac{A^{*}}{A}+\left(\alpha-\frac{1}{2} \sigma^{2}\right) \tau}{\sigma \sqrt{\tau}}\right) \\
& +\lim _{\sigma \rightarrow \infty}\left[\Phi\left(\frac{-\ln \frac{A^{*}}{A}-\left(\alpha-\frac{1}{2} \sigma^{2}\right) \tau}{\sigma \sqrt{\tau}}\right)\left(\frac{A^{*}}{A}\right)^{\frac{2 \alpha}{\sigma^{2}}-1}\right] \\
= & \lim _{\sigma \rightarrow \infty}\left[\Phi\left(\frac{-\ln \frac{A^{*}}{A}-\left(\alpha-\frac{1}{2} \sigma^{2}\right) \tau}{\sigma \sqrt{\tau}}\right)\left(\frac{A}{A^{*}}\right)\right] .
\end{aligned}
$$

Hence, if

$$
\lim _{\sigma \rightarrow \infty} A^{*}=\infty
$$

it holds that

$$
\lim _{\sigma \rightarrow \infty} P(T<\tau)=0 .
$$

We will show later that (A.29) holds for all relevant thresholds.

For $\tau<\tau^{*}$, the two effects work in opposite directions. As in the previous case, the mean $E[\tau]$ is increasing with uncertainty. Without a change in the volatility, an increase in 
the mean would then decrease the probability of replacing the existing production facility. However, increasing uncertainty results in a greater probability mass being present in the left tail of $\varphi(\tau)$. Therefore, the total effect of increasing uncertainty is ambiguous in this region. However, we are able to conclude that the probability of investing at a given $\tau$ behaves in a certain non-monotonic way. For $\sigma=0$, there is no probability mass on the interval $\left[0, \tau^{*}\right)$, since the investment takes place at $\tau<\tau^{*}$ with probability 1 . Therefore an increase in uncertainty initially leads to an increased probability of investment. For relatively large $\sigma$ the effect of moving the mean of the distribution to the right starts to dominate and the probability of asset replacement falls. For $\sigma \rightarrow \infty$ the probability of replacing the existing asset before a given time $\tau$ decreases to zero.

Finally, we show that all the thresholds increase with uncertainty monotonically and unboundedly. We already know (from Sections 5 and 6) that the optimal replacement thresholds increase with uncertainty monotonically. So now we only have to prove that the thresholds grow in uncertainty unboundedly. For the thresholds of the follower and in case of simultaneous replacement it is easy to observe that $\frac{\beta}{\beta-1}$ tends to infinity when $\sigma \rightarrow \infty .^{27}$ The replacement threshold of the leader requires slightly more attention. ${ }^{28}$ We already know that the leader replaces its asset as soon as the stochastic variable reaches the smallest root of the following equation (cf. (25))

$$
0=\frac{2 K A}{3 \delta}-\frac{K^{2}}{3 r}-I-\left(\frac{2 K A^{F}}{3 \delta}-\frac{K^{2}}{3 r}-I\right)\left(\frac{A}{A^{F}}\right)^{\beta}
$$

After substituting (16) into (A.30) and rearranging, we obtain that

$$
\begin{aligned}
0= & \left(1-\left(\frac{\frac{4}{9} K A}{\frac{\beta}{\beta-1}\left(I+\frac{4 K^{2}}{9 r}\right) \delta}\right)^{\beta-1}+\frac{\left(\frac{K^{2}}{3 r}+I\right) A^{\beta-1}}{\frac{2 K}{3 \delta}\left(\frac{\beta}{\beta-1} \frac{I+\frac{4 K^{2}}{9 r}}{\frac{4}{9} K A} \delta\right)^{\beta}}\right) \\
& \times \frac{2 K A}{3 \delta}-\frac{K^{2}}{3 r}-I .
\end{aligned}
$$

It holds that

$$
\lim _{\beta \downarrow 1}\left(1-\left(\frac{\frac{4}{9} K A}{\frac{\beta}{\beta-1}\left(I+\frac{4 K^{2}}{9 r}\right) \delta}\right)^{\beta-1}+\frac{\left(\frac{K^{2}}{3 r}+I\right) A^{\beta-1}}{\frac{2 K}{3 \delta}\left(\frac{\beta}{\beta-1} \frac{I+\frac{4 K^{2}}{9 r}}{\frac{4}{9} K A} \delta\right)^{\beta}}\right)=0 .
$$

\footnotetext{
${ }^{27}$ For a new market model a similar conclusion can be drawn after the substitution of parameters in the original geometric Brownian motion.

${ }^{28}$ The unboundedness of the leader threshold in the new market entry can be proven in a similar way as in the presented case of asset replacement.
} 
Since

$$
\lim _{\beta \downarrow 1} \frac{\partial}{\partial \beta}\left[1-\left(\frac{\frac{4}{9} K A}{\frac{\beta}{\beta-1}\left(I+\frac{4 K^{2}}{9 r}\right) \delta}\right)^{\beta-1}+\frac{\left(\frac{K^{2}}{3 r}+I\right) A^{\beta-1}}{\frac{2 K}{3 \delta}\left(\frac{\beta}{\beta-1} \frac{I+\frac{4 K^{2}}{9 r}}{\frac{4}{9} K A} \delta\right)^{\beta}}\right]>0,
$$

the LHS of (A.32) approaches zero from above. To shorten the notation we rewrite (A.31) into

$$
0=M(A) A-N
$$

Now, we are looking for the solution of (A.34). From (A.33) it can be seen that $m(A)$ is tending to zero from above $\forall A \in \mathbb{R}^{++}$when uncertainty is increasing. Consequently, any solution (so the smallest one as well) of (A.34) is tending to infinity. This is equivalent to

$$
\lim _{\beta \downarrow 1} A^{P}=\infty
$$

which completes the proof.

\section{B Limiting values of optimal thresholds to start production}

Limiting value of the optimal follower threshold. We are interested in the following limit

$$
\begin{aligned}
& \lim _{\sigma^{2} \rightarrow r-2 \alpha} 3 \sqrt{\frac{\beta}{\beta-2} I \varrho} \\
= & 3 \sqrt{\lim _{\sigma^{2} \rightarrow r-2 \alpha} \frac{\beta}{\beta-2} \varrho} .
\end{aligned}
$$

Furthermore, we have

$$
\begin{aligned}
& \lim _{\sigma^{2} \rightarrow r-2 \alpha} \frac{\beta}{\beta-2} \varrho \\
= & \lim _{\sigma^{2} \rightarrow r-2 \alpha} \frac{\left(\frac{1}{2}-\frac{\alpha}{\sigma^{2}}+\sqrt{\left.\left(\frac{\alpha}{\sigma^{2}}-\frac{1}{2}\right)^{2}+\frac{2 r}{\sigma^{2}}\right)}\left(r-2 \alpha-\sigma^{2}\right)\right.}{-\frac{3}{2}-\frac{\alpha}{\sigma^{2}}+\sqrt{\left(\frac{\alpha}{\sigma^{2}}-\frac{1}{2}\right)^{2}+\frac{2 r}{\sigma^{2}}}} \\
= & 2(r-2 \alpha) \lim _{\sigma^{2} \rightarrow r-2 \alpha} \frac{r-2 \alpha-\sigma^{2}}{-\frac{3}{2} \sigma^{2}-\alpha+\sqrt{\left(\alpha-\frac{\sigma^{2}}{2}\right)^{2}+2 r \sigma^{2}}} .
\end{aligned}
$$


Applying l'Hôpital's rule yields

$$
\begin{aligned}
& 2(r-2 \alpha) \lim _{\sigma^{2} \rightarrow r-2 \alpha-\frac{3}{2}+\frac{-\alpha+\frac{1}{2} \sigma^{2}+2 r}{2 \sqrt{\left(\alpha-\frac{\sigma^{2}}{2}\right)^{2}+2 r \sigma^{2}}}} \\
= & 2(r-2 \alpha) \frac{-1}{-\frac{3}{2}+\frac{-\alpha+\frac{1}{2}(r-2 \alpha)+2 r}{2 \sqrt{\left(\alpha-\frac{1}{2}(r-2 \alpha)\right)^{2}+2 r(r-2 \alpha)}}} \\
= & \frac{-4(r-2 \alpha) \sqrt{\left(\alpha-\frac{1}{2}(r-2 \alpha)\right)^{2}+2 r(r-2 \alpha)}}{-3 \sqrt{\left(\alpha-\frac{1}{2}(r-2 \alpha)\right)^{2}+2 r(r-2 \alpha)}-\alpha+\frac{1}{2}(r-2 \alpha)+2 r} \\
= & \frac{-4(r-2 \alpha)\left|2 \alpha-\frac{3}{2} r\right|}{-3\left|2 \alpha-\frac{3}{2} r\right|-\alpha+\frac{1}{2}(r-2 \alpha)+2 r} .
\end{aligned}
$$

Since $2 \alpha<r$, this is equal to

$$
\begin{aligned}
& \frac{4(r-2 \alpha)\left(2 \alpha-\frac{3}{2} r\right)}{3\left(2 \alpha-\frac{3}{2} r\right)-\alpha+\frac{1}{2}(r-2 \alpha)+2 r} \\
= & \frac{4(r-2 \alpha)\left(2 \alpha-\frac{3}{2} r\right)}{-2(r-2 \alpha)} \\
= & 3 r-4 \alpha .
\end{aligned}
$$

Substituting (B.2) into (B.1) yields the desired result.

Limiting value of the optimal leader threshold . To obtain the leader's limiting threshold, we are interested in the form of function $\xi^{N}$ when $\sigma^{2}$ tends to $r-2 \alpha$. For any $A \in\left(0, A^{F N}\right)$ we have (cf. (44), (45) and $\xi^{N}=V^{L N}-V^{F N}$ )

$$
\begin{aligned}
& \lim _{\sigma^{2} \rightarrow r-2 \alpha}\left[\frac{1}{4} \frac{A^{2}}{r-2 \alpha-\sigma^{2}}-I-\left(\frac{1}{4} \frac{\left(A^{F N}\right)^{2}}{r-2 \alpha-\sigma^{2}}-I\right)\left(\frac{A}{A^{F N}}\right)^{\beta}\right] \\
= & \lim _{\sigma^{2} \rightarrow r-2 \alpha}\left[\frac{1}{4} \frac{A^{2}}{r-2 \alpha-\sigma^{2}}-I-\left(\frac{1}{4} \frac{9(3 r-4 \alpha) I}{r-2 \alpha-\sigma^{2}}-I\right)\left(\frac{A}{3 \sqrt{(3 r-4 \alpha) I}}\right)^{\beta}\right],
\end{aligned}
$$

by using the limit of (41). Consequently, we rearrange (B.3) to get ${ }^{29}$

$$
\begin{aligned}
& \lim _{\sigma^{2} \rightarrow r-2 \alpha}\left[\frac{1}{4} \frac{A^{2}}{r-2 \alpha-\sigma^{2}}-I+\frac{A^{2}}{9(3 r-4 \alpha)}-\frac{1}{4} \frac{9(3 r-4 \alpha) I}{r-2 \alpha-\sigma^{2}}\left(\frac{A}{3 \sqrt{(3 r-4 \alpha) I}}\right)^{\beta}\right] \\
= & \frac{A^{2}}{9(3 r-4 \alpha)}-I+\frac{A^{2}}{4} \lim _{\sigma^{2} \rightarrow r-2 \alpha} \frac{1-\left(\frac{A}{3 \sqrt{(3 r-4 \alpha) I}}\right)^{\beta-2}}{r-2 \alpha-\sigma^{2}} .
\end{aligned}
$$

\footnotetext{
${ }^{29}$ We do so by observing that $\lim _{\sigma^{2} \rightarrow r-2 \alpha} \beta=2$.
} 
The limit in the last component can be calculated as follows:

$$
\lim _{\sigma^{2} \rightarrow r-2 \alpha} \frac{1-\left(\frac{A}{3 \sqrt{(3 r-4 \alpha) I}}\right)^{\beta-2}}{r-2 \alpha-\sigma^{2}}=\lim _{\sigma^{2} \rightarrow r-2 \alpha} \frac{1-\left(\frac{A}{3 \sqrt{(3 r-4 \alpha) I}}\right)^{-\frac{3}{2}-\frac{\alpha}{\sigma^{2}}+\sqrt{\left(\frac{\alpha}{\sigma^{2}}-\frac{1}{2}\right)^{2}+\frac{2 r}{\sigma^{2}}}}}{r-2 \alpha-\sigma^{2}} .
$$

Application of l'Hôpital's rule yields

$$
\begin{aligned}
& \lim _{\sigma^{2} \rightarrow r-2 \alpha} \frac{\partial}{\partial\left(\sigma^{2}\right)}\left(\left(\frac{A}{3 \sqrt{(3 r-4 \alpha) I}}\right)^{-\frac{3}{2}-\frac{\alpha}{\sigma^{2}}+\sqrt{\left(\frac{\alpha}{\sigma^{2}}-\frac{1}{2}\right)^{2}+\frac{2 r}{\sigma^{2}}}}\right) \\
= & \lim _{\sigma^{2} \rightarrow r-2 \alpha} \ln \left(\frac{A}{3 \sqrt{(3 r-4 \alpha) I}}\right)\left(\frac{A}{3 \sqrt{(3 r-4 \alpha) I}}\right)^{-\frac{3}{2}-\frac{\alpha}{\sigma^{2}}+\sqrt{\left(\frac{\alpha}{\sigma^{2}}-\frac{1}{2}\right)^{2}+\frac{2 r}{\sigma^{2}}}} \\
& \times\left(\frac{\alpha}{\sigma^{4}}-\frac{\frac{\alpha}{\sigma^{4}}\left(\frac{\alpha}{\sigma^{2}}-\frac{1}{2}\right)+\frac{r}{\sigma^{4}}}{\sqrt{\left(\frac{\alpha}{\sigma^{2}}-\frac{1}{2}\right)^{2}+\frac{2 r}{\sigma^{2}}}}\right) \\
= & \ln \left(\frac{A}{3 \sqrt{(3 r-4 \alpha) I}}\right)\left(\frac{\alpha}{(r-2 \alpha)^{2}}-\frac{\frac{\alpha}{r-2 \alpha}\left(\frac{\alpha}{r-2 \alpha}-\frac{1}{2}\right)+\frac{r}{r-2 \alpha}}{\frac{3}{2} r-2 \alpha}\right) \\
= & \ln \left(\frac{A}{3 \sqrt{(3 r-4 \alpha) I}}\right)\left(-\frac{1}{\frac{3}{2} r-2 \alpha}\right) .
\end{aligned}
$$

Consequently, after substituting (B.7) into (B.4), we obtain the formula for the limiting case of $\xi^{N}$ :

$$
\left(\left(\frac{A}{A^{F N}}\right)^{2}-1\right) I-\frac{A^{2}}{2(3 r-4 \alpha)} \ln \left(\frac{A}{A^{F N}}\right)
$$




\section{References}

Bodie Z, Kane A, Marcus AJ (2002) Investments, 5th printing. McGraw-Hill Irwin, New York

Boyer M, Lasserre P, Mariotti T, Moreaux M (2002) Real options, preemption, and the dynamics of industry investments. Working Paper, CIRANO and University of Toulouse

Bower JL (1986) Managing the resource allocation process. Harvard Business School Classics, Cambridge MA

Bulan LT (2001) Real options, irreversible investment, and firm uncertainty. Working Paper, Brandeis University

Caballero RJ, Pindyck RS (1996) Uncertainty, investment and industry evolution. International Economic Review 37 : 641-662

Dixit A (1993) The art of smooth pasting. Vol. 55 in Fundamentals of Pure and Applied Economics, Harwood Academic Publishers, New York

Dixit A, Pindyck RS (1995) The options approach to capital investment. Harvard Business Review 73 : 105-118

Dixit A, Pindyck RS (1996) Investment under Uncertainty, 2nd printing. Princeton University Press, Princeton NJ

Fama EF, French KR (2001) Disappearing dividends: changing firm characteristics or lower propensity to pay? Journal of Financial Economics $60: 3-43$

Fudenberg D, Tirole J (1985) Preemption and rent equalization in the adoption of new technology. Review of Economic Studies $52: 383-401$

Grenadier SR (1996) The strategic exercise of options: Development cascades and overbuilding in real estate markets. Journal of Finance 51 : 1653-1679

Harrison MJ (1985) Brownian motion and stochastic flow systems. John Wiley \& Sons, New York

Harsanyi JC (1973) Games with randomly distributed payoffs: A new rationale for mixed strategy equilibrium points. International Journal of Game Theory $2: 1-13$

Holland AS, Ott SH, Riddiough TJ (2000) The role of uncertainty in investment: An examination of competing investment models using commercial real estate data. Real Estate Economics $28: 33-64$

Hoppe H, Lehmann-Grube U (2001) Second-mover advantages in dynamic quality competition. Journal of Economics and Management Strategy 10 : 419-433 
Huisman KJM, Kort PM (1999) Effects of strategic interactions on the option value of waiting. CentER DP 9982, Tilburg University

Kaplan RS, Atkinson AA (1998) Advanced management accounting, 3rd edition. Prentice Hall, Upper Saddle River NJ

Kester WC (1984) Today's options for tomorrow's growth. Harvard Business Review 62 : 105-118

Kulatilaka N, Perotti EC (1998) Strategic growth options. Management Science 44 : 10211031

McDonald R, Siegel DR (1986) The value of waiting to invest. Quarterly Journal of Economics $101: 707-728$

Milgrom P, Weber R (1986) Distributional strategies for games with incomplete information. Mathematics of Operations Research $10: 619-631$

Myers SC (1987) Finance theory and financial strategy. Midland Corporate Finance Journal $5: 6-13$

Paddock JL, Siegel DR, Smith JL (1988) Option valuation of claims on real assets: The case of offshore petroleum leases. Quarterly Journal of Economics 103 : 755-784

Pawlina G, Kort PM (2002) Real options in an asymmetric duopoly: Who benefits from your competitive disadvantage? Working Paper (updated version of CentER DP 2001-95), Tilburg University

Pennings E (2002) Optimal pricing and quality choice when investment in quality is irreversible. Working Paper, IGIER - Bocconi University

Perotti EC, Rossetto S (2000) Internet portals as portfolios of entry options. Working Paper, University of Amsterdam

Quigg L (1993) Empirical testing of real option pricing models. Journal of Finance 44 : 1115-1153

Reinganum JF (1981) On the diffusion of new technology: A game theoretic approach. Review of Economic Studies 48 : 395-405

Sarkar S (2000) On the investment-uncertainty relationship in a real options model. Journal of Economic Dynamics and Control 24 : 219-225

Smets F (1991) Exporting versus FDI: The effect of uncertainty irreversibilities and strategic interactions. Working Paper, Yale University

Shapiro C, Varian HR (1998) Information rules. Harvard Business School Press, Cambridge MA 
Thijssen JJJ, Huisman KJM, Kort PM (2002) Symmetric equilibrium strategies in game theoretic real option models. CentER DP 2002-81, Tilburg University

Torvund PA (1999) Strategic timing behaviour and related game theoretical topics. Ph.D. Thesis, Department of Mathematics, University of Oslo

Trigeorgis L (1988) A conceptual options framework for capital budgeting. Advances in Futures and Options Research $3: 145-167$

Trigeorgis L, Mason SP (1988) Valuing managerial flexibility. Midland Corporate Finance Journal $5: 14-21$

Tirole J (1988). The theory of industrial organization, MIT Press, Cambridge MA

Zingales L (2000) In search of new foundations. Journal of Finance 55 : 1623-1653 